CTP TAMU-26/96

DAMTP-R/96/32

Imperial/TP/95-96/68

SISSA $124 / 96 / \mathrm{EP}$

hep-th/9608173

\title{
Domain Walls in Massive Supergravities
}

\author{
P.M. Cowdall ${ }^{(1)}$, H. Lü ${ }^{(2,3)}$, C.N. Pope ${ }^{(2,3, \dagger)}$, K.S. Stelle ${ }^{(4, \star)}$ and P.K. Townsend ${ }^{(1)}$ \\ (1) DAMTP, University of Cambridge, Silver St., Cambridge CB3 9EW, U.K. \\ (2) Center for Theoretical Physics, Texas A\&M University, College Station, Texas 77843 \\ (3) SISSA, Via Beirut No. 2-4, 34013 Trieste, Italy \\ (4) The Blackett Laboratory, Imperial College, Prince Consort Road, London SW7 2BZ
}

\begin{abstract}
$\underline{\text { ABSTRACT }}$
We show how toroidally-compactified eleven-dimensional supergravity can be consistently truncated to yield a variety of maximally-supersymmetric massive supergravities in spacetime dimensions $D \leq 8$. The mass terms arise as a consequence of making a more general ansatz than that in usual Kaluza-Klein dimensional reduction, in which one or more axions are given an additional linear dependence on one of the compactification coordinates. The lower-dimensional theories are nevertheless consistent truncations of eleven-dimensional supergravity. Owing to the fact that the generalised reduction commutes neither with Uduality nor with ordinary dimensional reduction, many different massive theories can result. The simplest examples arise when just a single axion has the additional linear coordinate dependence. We find five inequivalent such theories in $D=7$, and 71 inequivalent ones in $D=4$. The massive theories admit no maximally-symmetric vacuum solution, but they do admit $(D-2)$-brane solutions, i.e. domain walls, which preserve half the supersymmetry. We present examples of these solutions, and their oxidations to $D=11$. Some of the latter are new solutions of $D=11$ supergravity.
\end{abstract}

\footnotetext{
${ }^{\dagger}$ Research supported in part by DOE Grant DE-FG05-91-ER40633 and EC Human Capital and Mobility Programme under contract ERBCHBGCT920176.

* Research supported in part by the Commission of the European Communities under contract SCI*-CT92-0789.
} 


\section{Introduction}

M-theory or string theory, and their dimensional reductions, admit a multitude of $p$-brane soliton solutions. These can be understood from a variety of perspectives. In particular, one way to classify them is by giving their explicit construction in the various lowerdimensional supergravities obtained by dimensional reduction from M-theory or string theory. An isotropic $p$-brane solution in $D$ dimensions is supported by either an electric or a magnetic charge carried by an antisymmetric-tensor field strength in the theory; a rank $p+2$ tensor in the electric case, or a rank $(D-p-2)$ tensor in the magnetic case. The numbers of field strengths with ranks $\leq 3$ grow rapidly with decreasing $D$, and, correspondingly, the numbers of $p$-brane solutions for each $p$ increase also. These multiplicities of solutions are classified by the $\mathrm{U}$ duality symmetries of the corresponding supergravity theories, (or more precisely, by the Weyl groups of the U duality groups [1]). Another approach to the classification of $p$-brane solitons is to follow their evolution through the various steps of dimensional reduction [2], from a set of higher-dimensional solutions of the original M-theory or string theory. Some, but not all, of these higher-dimensional solutions are themselves $p$-brane solitons.

An essential ingredient that allows one to give these two kinds of description for the $p$-brane spectrum is the fact that the Kaluza-Klein reduction of the higher-dimensional theory is a consistent truncation. In particular, this means that all solutions of the lowerdimensional theory are necessarily also solutions of the higher-dimensional one. A further feature is that (down to $p=D-3$, as we shall see), from a given starting-point in the higher dimension, there is a unique theory relevant to the solutions in the lower dimension. This may, without loss of generality, be taken to be the maximal supergravity in that dimension, as all lesser supergravities may be taken to be consistent truncations of a maximal theory. This unique theory has a high degree of symmetry, namely the U duality referred to above, which incorporates the multiplicity of reduction routes one may use to arrive at a given pair $(D, d)$ of spacetime and worldvolume dimensions.

These different routes employ two basic types of reduction of $p$-brane solutions. The first of the two reduction procedures makes use of the Poincaré symmetry of such solutions on the $p$-brane worldvolumes, and results in a simultaneous reduction of the spacetime and worldvolume dimensions, from $(D, d)$ to $(D-1, d-1)$ [4, 35, 5n. The second reduction procedure involves first constructing a "stack" of parallel $p$-branes. By taking the continuum limit as this stack becomes densely packed, a translational invariance is generated in a direction transverse to the worldvolume of the $p$-brane, and this can then be used for the 
Kaluza-Klein reduction [6, 2]. In this case, $(D, d)$ is reduced to $(D-1, d)$. The two reduction procedures have been called "diagonal" and "vertical," respectively. In performing a vertical reduction, taking the continuum limit of a stack of $p$-branes amounts to replacing a sum by an integral within a multi-center solution of Laplace's equation in the transverse space, effectively reducing by one the dimension of the space in which the Laplacian acts. Thus, under vertical reduction, $(D, d)$ is reduced to $(D-1, d)$.

This process of generating harmonic functions in lower transverse dimensions continues uneventfully until one makes the reduction from the harmonic function $1 / r$ in three dimensions to $\log r$ in two dimensions, corresponding to the reduction from a $(D, D-3)$ solution to a $(D-1, D-3)$ solution. This step has two related features. The first is that the integral $\int_{-L}^{L} d z\left(r^{2}+z^{2}\right)^{-1 / 2}$ producing the $\log r$ harmonic function gives rise to an additive $2 \log L$ divergence as $L \rightarrow \infty$ that needs to be renormalised away. The second feature is that the $\log r$ harmonic function no longer dies off at transverse infinity. Its derivative, which governs the form of the field strength supporting the solution, still does fall off at transverse infinity, however.

Continuing on through one more step, the reduction from a two-dimensional to a onedimensional transverse space involves the integral $\int_{-L}^{L} d z \log \left(y^{2}+z^{2}\right)$ which again produces an additive divergence, this time of the form $4 L \log L-4 L$. After renormalising this away, the resulting harmonic function $H(y)$ is linear in the one remaining transverse-space coordinate $y$. Not only does this not vanish at transverse infinity, but its derivative is now a non-vanishing constant as well. This has the consequence that the corresponding 1-form field strength supporting the continuous stack of $(D-3)$-branes has the form (still in the two transverse dimensions) $F_{m}=-\epsilon_{m n} \partial_{n} H(y)$, and thus $F_{z}$, its component in the compactification direction, is a constant. This is a new phenomenon in the process of vertical reduction, which we shall study in detail in the rest of this paper. In particular, this has the consequence that the 0-form potential, or "axion," for the field strength $F_{m}$ must itself be linearly dependent on $z$. (The occurrence of membrane solutions in $D=4$ resulting from vertical reduction of a 5-brane of the heterotic string wrapped around a 3-torus was observed in []], where it was also noted that the usual Kaluza-Klein ansatz is not sufficient, since it does not give rise to the necessary cosmological term in the reduced $D=4$ theory.)

Normally, in Kaluza-Klein dimensional reduction, all of the higher-dimensional fields

\footnotetext{
${ }^{1}$ We shall refer generally to solutions of this latter sort, where the worldvolume dimension $d=p+1$ is 2 less than the spacetime dimension as "( $D-3)$-branes," regardless of whether one is in $D$ spacetime dimensions or not.
} 
are taken to be independent of the compactification coordinate $z$. Indeed, consistency of the truncation is assured by retaining all fields subject to this restriction. At first sight, one might think that a consistent truncation would no longer be possible if any dependence on the compactification coordinate were retained. However, the supergravity theories whose dimensional reduction we are considering have a special feature that the axionic field that is given the above $z$ dependence always appears in the Lagrangian covered by a derivative. Consequently, after insertion of the ansatz, the higher-dimensional Lagrangian and also the equations of motion will still be independent of $z$, thus permitting a consistent truncation even when we allow a more general Kaluza-Klein ansatz in which such an axion has a linear $z$ dependence. In fact, what vertical reduction has produced in this case is an instance of Scherk-Schwarz type Kaluza-Klein reduction [8, 9].2] However, unlike the original ScherkSchwarz procedure, which was designed to give a mass to the gravitino, thus breaking supersymmetry, it turns out in the present case that supersymmetry remains unbroken, but that certain bosonic fields become massive instead. The set of massive fields always includes the Kaluza-Klein vector potential arising from the reduction of the metric, the additional degree of freedom being supplied by the dimensionally-reduced axion that was given the linear $z$ dependence in the higher dimension. At the same time, a cosmologicaltype term is generated in the lower-dimensional theory, having the general form of $\sqrt{-g}$ times an exponential of dilatonic scalar fields. The first example of this kind of ScherkSchwarz procedure arose in the dimensional reduction of the type IIB theory [9], where it was shown that the resulting nine-dimensional theory is related by $T$ duality to the ordinary Kaluza-Klein reduction of massive IIA supergravity [10].

In the rest of this paper, we shall study the above process of Scherk-Schwarz dimensional reduction in supergravity theories, and the consequences of this for $p$-brane solutions. In doing so, our principal focus will be on the consistent reductions of the theories themselves, rather than on the relations between solutions of the reduced theories and solutions in higher dimensions. A particularly compelling reason for placing the emphasis on theories rather than on solutions is that, as we shall show, the process of Scherk-Schwarz dimensional reduction of a given theory can be done in as many ways as there are axions in the higherdimensional theory. This situation is to be contrasted with that prevailing for $p$-branes

\footnotetext{
${ }^{2}$ This instance of the Scherk-Schwarz procedure uses the shift symmetry of the selected axionic field as the global symmetry to be supplied with a $z$-dependent gauge parameter in the Kaluza-Klein reduction.

${ }^{3}$ We are using the term "axion" here to denote any scalar field that has a shift symmetry under which all other fields are inert, and thus can, after appropriate field redefinitions and integrations by parts, be covered everywhere by a derivative. The axion fields in the supergravity theories that we are considering all arise as
} 
with $p \leq(D-3)$, where there is always an essentially unique reduced supergravity theory (i.e. unique up to standard duality transformations). The various pathways of dimensional reduction above this $p=(D-3)$ "barrier" thus give rise to a multiplicity of different solutions to a single theory, with the multiplicity being classified by the Weyl group of the U-duality symmetry. Penetrating this $p=(D-3)$ barrier by the various forms of ScherkSchwarz reduction that we shall discuss gives rise, not to a multiplicity of solutions within a given theory, but to a multiplicity of different reduced theories (with only a trivial $Z_{2}$ residual classifying symmetry for the resulting $(D-2)$-brane solutions so obtained).

In the next section, we shall describe in detail the process of Scherk-Schwarz dimensional reduction, showing that it commutes neither with ordinary Kaluza-Klein reduction, nor with the U-duality symmetries. This latter point has the consequence that every axionic scalar gives rise to its own distinct Scherk-Schwarz reduced theory. Furthermore, the non-commutativity with ordinary Kaluza-Klein reduction means that the total number of distinct massive supergravities obtained by these procedures is the cumulative total of those already obtained in one higher dimension, plus the further examples obtained by ScherkSchwarz reduction in the final step. We shall discuss in detail the massive theories in the specific cases of $D=8$ and $D=7$, showing that there are two such distinct massive theories in $D=8$, and six distinct theories in $D=7$.

\section{Scherk-Schwarz dimensional reduction}

The highest-dimensional example of a massive supergravity theory is the massive IIA theory in $D=10$, which was constructed in [10]. It was shown in 9] that its Kaluza-Klein reduction to $D=9$ coincides, up to a $T$-duality transformation, with the massive theory obtained by Scherk-Schwarz reduction of the type IIB theory. Subsequent steps of Kaluza-Klein reduction will give rise to a single massive supergravity in each lower dimension. None of these examples can be obtained by any combination of Scherk-Schwarz and Kaluza-Klein reductions of $D=11$ supergravity. All other examples of massive supergravities of the kind that we are considering in this paper can, however, be obtained from $D=11$. Accordingly, we shall present just the cases derived from the $D=11$ theory in detail, concentrating principally on the bosonic sectors of the theories.

It is convenient to parametrise the standard Kaluza-Klein dimensional reductions of

the 0 -form potential endpoints of dimensional reductions of higher-rank potentials (except for the axion in type IIB supergravity). 
$D=11$ supergravity using the formalism presented in [11], which arrives at $D$ dimensions by means of a succession of 1-step reductions, rather than by a direct descent from 11 to $D$ dimensions. The usual Kaluza-Klein reduction procedure involves taking the higherdimensional fields to be independent of the compactification coordinate $z$. A convenient parametrisation for the metric and for an $n$-rank potential $A_{n}$, is then as follows:

$$
\begin{aligned}
d s^{2}(x, z) & \longrightarrow e^{2 \alpha \varphi(x)} d s^{2}(x)+e^{-2(D-2) \alpha \varphi(x)}\left(d z+\mathcal{A}_{M}(x) d x^{M}\right)^{2} \\
A_{n}(x, z) & \longrightarrow A_{n}(x)+A_{n-1}(x) \wedge d z
\end{aligned}
$$

where the fields in $(D+1)$ dimensions on the left are reduced to fields in $D$ dimensions on the right. The constant $\alpha$ is given by $\alpha=(2(D-1)(D-2))^{-1 / 2}$. This parametrisation of the metric is chosen so that the Einstein action $\sqrt{-g} R$ in the higher dimension reduces to $\sqrt{-g} R$ in the lower dimension. The field strengths are best expressed in terms of their orthonormal-frame components, obtained by contracting with vielbeins, since these most easily allow the forms of their lower-dimensional kinetic terms to be read off. Thus we find that a field strength $F_{n+1}(x, z)=d A_{n}(x, z)$ in the higher dimension reduces to

$$
d A_{n}(x)-d A_{n-1}(x) \wedge \mathcal{A}(x)+d A_{n-1}(x) \wedge(d z+\mathcal{A}) \equiv F_{n+1}(x)+F_{n}(x) \wedge(d z+\mathcal{A}),
$$

showing how Chern-Simons modifications of the form $F_{n+1}=d A_{n}-d A_{n-1} \wedge \mathcal{A}$ are developed as the reduction proceeds. The resulting bosonic Lagrangian for maximal supergravity in $D$ dimensions was obtained using this formalism in [11], and is presented in the Appendix.

In the context of $D=11$ supergravity reductions that we are considering, the highest dimension in which there is an axion available for performing a Scherk-Schwarz reduction is $D=9$. We shall begin by considering this example in some detail.

\subsection{Scherk-Schwarz reduction of $D=9$ supergravity}

From the formulae collected in the Appendix, the Lagrangian for massless $D=9$ maximal supergravity is given by

$$
\begin{aligned}
\mathcal{L}= & e R-\frac{1}{2} e\left(\partial \phi_{1}\right)^{2}-\frac{1}{2} e\left(\partial \phi_{2}\right)^{2}-\frac{1}{2} e e^{\phi_{1}+\frac{3}{\sqrt{7}} \phi_{2}}(\partial \chi)^{2}-\frac{1}{48} e e^{\vec{a} \cdot \vec{\phi}}\left(F_{4}\right)^{2} \\
& -\frac{1}{2} e e^{\vec{a}_{1} \cdot \vec{\phi}}\left(F_{3}^{(1)}\right)^{2}-\frac{1}{2} e e^{\vec{a}_{2} \cdot \vec{\phi}}\left(F_{3}^{(2)}\right)^{2}-\frac{1}{4} e e^{\vec{a}_{12} \cdot \vec{\phi}}\left(F_{2}^{(12)}\right)^{2}-\frac{1}{4} e e^{\overrightarrow{b_{1}} \cdot \vec{\phi}}\left(\mathcal{F}_{2}^{(1)}\right)^{2} \\
& -\frac{1}{4} e e^{\vec{b}_{2} \cdot \vec{\phi}}\left(\mathcal{F}_{2}^{(2)}\right)^{2}-\frac{1}{2} \tilde{F}_{4} \wedge \tilde{F}_{4} \wedge A_{1}^{(12)}-\tilde{F}_{3}^{(1)} \wedge \tilde{F}_{3}^{(2)} \wedge A_{3},
\end{aligned}
$$

where we have defined $\vec{\phi}=\left(\phi_{1}, \phi_{2}\right)$, and $\chi=\mathcal{A}_{0}^{(12)}$. We have made the dilaton coupling within the scalar-manifold sector explicit, while in the rest of the Lagrangian the dilaton 
vectors are as given in the Appendix. The Chern-Simons modifications to the field strengths are given by

$$
\begin{aligned}
F_{4} & =\tilde{F}_{4}-\tilde{F}_{3}^{(1)} \wedge \mathcal{A}_{1}^{(1)}-\tilde{F}_{3}^{(2)} \wedge \mathcal{A}_{1}^{(2)}+\chi \tilde{F}_{3}^{(1)} \wedge \mathcal{A}_{1}^{(2)}-\tilde{F}_{2}^{(12)} \wedge \mathcal{A}_{1}^{(1)} \wedge \mathcal{A}_{1}^{(2)}, \\
F_{3}^{(1)} & =\tilde{F}_{3}^{(1)}-\tilde{F}_{2}^{(12)} \wedge \mathcal{A}_{1}^{(2)} \\
F_{3}^{(2)} & =\tilde{F}_{3}^{(2)}+F_{2}^{(12)} \wedge \mathcal{A}_{1}^{(1)}-\chi \tilde{F}_{3}^{(1)}, \\
F_{2}^{(12)} & =\tilde{F}_{2}^{(12)}, \quad \mathcal{F}_{2}^{(1)}=\mathcal{F}_{2}^{(1)}-d \chi \wedge \mathcal{A}_{1}^{(2)}, \quad \mathcal{F}_{2}^{(2)}=\tilde{\mathcal{F}}_{2}^{(2)}, \quad \mathcal{F}_{1}^{(12)}=d \chi .
\end{aligned}
$$

As it stands, we can see from (2.4) that the axion $\chi$ appears without yet being covered by a derivative in certain places in the Lagrangian. However, it is easy to remedy this by performing the following field redefinition:

$$
A_{2}^{(2)} \longrightarrow A_{2}^{(2)}+\chi A_{2}^{(1)}
$$

after which the Chern-Simons modifications (2.4) become

$$
\begin{aligned}
F_{4} & =\tilde{F}_{4}-\tilde{F}_{3}^{(1)} \wedge \mathcal{A}_{1}^{(1)}-\tilde{F}_{3}^{(2)} \wedge \mathcal{A}_{1}^{(2)}-d \chi \wedge A_{2}^{(1)} \wedge \mathcal{A}_{1}^{(2)}-\tilde{F}_{2}^{(12)} \wedge \mathcal{A}_{1}^{(1)} \wedge \mathcal{A}_{1}^{(2)}, \\
F_{3}^{(1)} & =\tilde{F}_{3}^{(1)}-\tilde{F}_{2}^{(12)} \wedge \mathcal{A}_{1}^{(2)} \\
F_{3}^{(2)} & =\tilde{F}_{3}^{(2)}+F_{2}^{(12)} \wedge \mathcal{A}_{1}^{(1)}+d \chi \wedge A_{2}^{(1)}, \\
F_{2}^{(12)} & =\tilde{F}_{2}^{(12)}, \quad \mathcal{F}_{2}^{(1)}=\mathcal{F}_{2}^{(1)}-d \chi \wedge \mathcal{A}_{1}^{(2)}, \quad \mathcal{F}_{2}^{(2)}=\tilde{\mathcal{F}}_{2}^{(2)}, \quad \mathcal{F}_{1}^{(12)}=d \chi .
\end{aligned}
$$

One may argue on general grounds that, having established that the axion $\chi$ can be covered by a derivative everywhere in the bosonic sector, the same will also be true in the fermionic sector. The reason for this is that the full set of scalar fields in maximal supergravity (i.e. both axions and dilatons) are described by a coset manifold, and that their couplings to the fermions occur via the vielbein on this coset manifold. We have seen above that the metric on the coset manifold can be cast into a form where there is no dependence on the axion $\chi$, and therefore it follows that a vielbein basis with this same property can also be chosen. Then, the entire supergravity Lagrangian will involve $\chi$ covered everywhere by a derivative.

We are now in a position to proceed with the Scherk-Schwarz dimensional reduction to $D=8$. Denoting the compactification coordinate by $z$, we make the usual Kaluza-Klein ansatz (2.1) that all the lower-dimensional fields are independent of $z$, with the exception of the axion $\chi$, for which the ansatz will be

$$
\chi(x, z) \longrightarrow m z+\chi(x)
$$

Note that the vielbein components of the field strength for $\chi$ can then be read off from $d \chi \rightarrow d \chi-m \mathcal{A}+m(d z+\mathcal{A})$, implying in particular that there will be a "0-form field 
strength" $m$, which will give rise to a cosmological-type term in the lower dimension. It is manifest that the higher-dimensional equations of motion, after substitution of the ansatz, will be independent of $z$, and hence it follows that this truncation to $D=8$ will be consistent, since all the $z$-independent degrees of freedom are being retained. Furthermore, it follows for the same reason that the $D=8$ equations of motion are derivable from the $D=8$ Lagrangian that one obtains by simply substituting the ansatz into the $D=9$ Lagrangian.

Performing the substitution of the ansatz into (2.3), we obtain the following $D=8$ Lagrangian:

$$
\begin{aligned}
e^{-1} \mathcal{L}= & R-\frac{1}{2}\left(\partial \phi_{1}\right)^{2}-\frac{1}{2}\left(\partial \phi_{2}\right)^{2}-\frac{1}{2}\left(\partial \phi_{3}\right)^{2}-\frac{1}{2} e^{\vec{b}_{12} \cdot \vec{\phi}}\left(\partial \chi-m \mathcal{A}_{1}^{(3)}\right)^{2} \\
& -\frac{1}{2} e^{\vec{b}_{13} \cdot \vec{\phi}}\left(\partial \mathcal{A}_{0}^{(13)}+m \mathcal{A}_{1}^{(2)}\right)^{2}-\frac{1}{2} e^{\vec{b}_{23} \cdot \vec{\phi}}\left(\partial \mathcal{A}_{0}^{(23)}\right)^{2}-\frac{1}{2} e^{\vec{a}_{123} \cdot \vec{\phi}}\left(\partial A_{0}^{(123)}\right)^{2} \\
& -\frac{1}{48} e^{\vec{a} \cdot \vec{\phi}}\left(F_{4}-m A_{2}^{(1)} \wedge \mathcal{A}_{1}^{(2)} \wedge \mathcal{A}_{1}^{(3)}\right)^{2}-\frac{1}{12} e^{\vec{a}_{1} \cdot \vec{\phi}}\left(F_{3}^{(1)}\right)^{2} \\
& -\frac{1}{12} e^{\vec{a}_{2} \cdot \vec{\phi}}\left(F_{3}^{(2)}+m A_{2}^{(1)} \wedge \mathcal{A}_{1}^{(3)}\right)^{2}-\frac{1}{12} e^{\vec{a}_{3} \cdot \vec{\phi}}\left(F_{3}^{(3)}+m A_{2}^{(1)} \wedge \mathcal{A}_{1}^{(2)}\right)^{2} \\
& -\frac{1}{4} e^{\vec{a}_{12} \cdot \vec{\phi}}\left(F_{2}^{(12)}\right)^{2}-\frac{1}{4} e^{\vec{a}_{13} \cdot \vec{\phi}}\left(F_{2}^{(13)}\right)^{2}-\frac{1}{4} e^{\vec{a}_{23} \cdot \vec{\phi}}\left(F_{2}^{(23)}+m A_{2}^{(1)}\right)^{2} \\
& -\frac{1}{4} e^{\vec{b}_{1} \cdot \vec{\phi}}\left(\mathcal{F}_{2}^{(1)}-m \mathcal{A}_{1}^{(2)} \wedge \mathcal{A}_{1}^{(3)}\right)^{2}-\frac{1}{4} e^{\vec{b}_{2} \cdot \vec{\phi}}\left(\mathcal{F}_{2}^{(2)}\right)^{2}-\frac{1}{4} e^{\vec{b}_{3} \cdot \vec{\phi}}\left(\mathcal{F}_{2}^{(3)}\right)^{2} \\
& -\frac{1}{2} m^{2} e^{\vec{b}_{123} \cdot \vec{\phi}}+e^{-1} \mathcal{L}_{F F A}
\end{aligned}
$$

where $\mathcal{L}_{F F A}$ is the same as the term given in (A.8) for massless $D=8$ supergravity, and the dilaton vectors are given by (A.2) for the case $D=8$. The Chern-Simons modifications for the field strengths appearing in (2.8) are essentially those given by (2.4) for the massless $D=8$ theory, after taking into account the field redefinition (2.5). The penultimate term in (2.8) is a cosmological-type term, whose dilaton vector $\vec{b}_{123}$ is given in (A.2).

It is now apparent that some of the fields in the $D=8$ theory that we have obtained by Scherk-Schwarz reduction have acquired masses. Specifically, we see that after performing the following gauge transformations:

$$
\begin{aligned}
& \mathcal{A}_{1}^{(3)} \longrightarrow \mathcal{A}_{1}^{(3)}+\frac{1}{m} d \chi \\
& \mathcal{A}_{1}^{(2)} \longrightarrow \mathcal{A}_{1}^{(2)}-\frac{1}{m} d \mathcal{A}_{0}^{(13)} \\
& A_{2}^{(1)} \longrightarrow A_{2}^{(1)}-\frac{1}{m} d A_{1}^{(23)}
\end{aligned}
$$

the fields $\mathcal{A}_{1}^{(3)}, \mathcal{A}_{1}^{(2)}$ and $A_{2}^{(1)}$ absorb the fields $\chi, \mathcal{A}_{0}^{(13)}$ and $A_{1}^{(23)}$ respectively. Thus $\mathcal{A}_{1}^{(3)}$, $\mathcal{A}_{1}^{(2)}$ and $A_{2}^{(1)}$ become massive in the Scherk-Schwarz reduction process.

This example of Scherk-Schwarz reduction from $D=9$ to $D=8$ illustrates the general pattern of mass generation. Firstly, the Kaluza-Klein vector potential arising in the reduction $\left(\mathcal{A}_{1}^{(3)}\right.$ in this example) always acquires a mass, absorbing the dimensional reduction of 
the axion that was used in the Scherk-Schwarz reduction. Secondly, if this axion appears in any bilinear term in the Chern-Simons modifications for any of the higher-dimensional fields e.g. $H=d B+d \chi \wedge A+\cdots$, then the corresponding field $A$ acquires a mass also, by absorbing the field $C$ obtained from the dimensional reduction of $B, B(x, z) \rightarrow B(x)+C(x) \wedge d z$. There is also a third kind of mass generation that can arise, which is not present in the above example. This can happen if the axion that is used in the Scherk-Schwarz reduction is one of the $A_{0}^{(i j k)}$ fields coming from the dimensional reduction of the 3-form potential in $D=11$. In this case, we see from (A.8) that it will give rise to a kind of topological mass term in the lower dimension. The first example where such an axion exists is in $D=8$, and we shall discuss this case in more detail below.

It is worth emphasising that the massive theory in $D=8$ that we have obtained above is quite distinct from the $D=8$ massive theory that is obtained by performing a standard Kaluza-Klein reduction of the massive IIA theory occurring in $D=10$ 10. In this $D=10$ theory, the only massive field is a 3-form field strength. Upon performing two steps of Kaluza-Klein reduction, this gives one massive 3-form, two massive 2-forms, and one massive 1 -form in $D=8$. By contrast, the theory described by (2.8) has only a massive 3 -form and two massive 2-form field strengths. Thus the two theories are manifestly inequivalent.

This inequivalence of the two $D=8$ massive theories illustrates another important feature of the Scherk-Schwarz reduction procedure, namely that it does not commute with ordinary Kaluza-Klein reduction. To see this, we note that we could alternatively have obtained the theory (2.8) by performing first an ordinary Kaluza-Klein reduction of the type IIB theory, followed by a Scherk-Schwarz reduction step. On the other hand, the two-step reduction of the massive IIA theory could alternatively have been obtained by performing first a Scherk-Schwarz reduction of the IIB theory [9], followed by an ordinary Kaluza-Klein reduction step. The inequivalence of the two massive theories in $D=8$ thus demonstrates the non-commutativity of the Scherk-Schwarz and ordinary Kaluza-Klein reductions.

It is of interest also to examine the surviving symmetries in the Scherk-Schwarz reduced theory. Under ordinary Kaluza-Klein reduction, the $G L(2, \mathbb{R})$ Cremmer-Julia symmetry group in $D=9$ enlarges to $S L(2, \mathbb{R}) \times S L(3, \mathbb{R})$ in $D=8[15]$. Since the Scherk-Schwarz procedure gives a non-vanishing constant value to the internal component of the 1-form field strength $\mathcal{F}_{1}^{(12)}$, we can expect that the surviving symmetry group in $D=8$ will be smaller than $S L(2, \mathbb{R}) \times S L(3, \mathbb{R})$. As in the massless theory cases, we can study this symmetry by looking at its action on the scalar manifold describing the dilatonic and axionic sector of the theory. In particular, we can see from (2.8) that the residual symmetry 
transformations must leave the cosmological term invariant, and thus the component of the dilatonic scalars $\vec{\phi}$ that is parallel to $\vec{b}_{123}$ must be inert. Now we find that $\vec{b}_{123} \cdot \vec{b}_{23}=0$, while $\vec{b}_{123} \cdot \vec{a}_{123} \neq 0$. It therefore follows that there is an $S L(2, \mathbb{R})$ invariance involving the axion $\mathcal{A}_{0}^{(23)}$, together with the linear combination of dilatons $\varphi \equiv-\frac{1}{2} \vec{b}_{23} \cdot \vec{\phi}$, since they are described simply by the terms $-\frac{1}{2}(\partial \varphi)^{2}-\frac{1}{2} e^{-2 \varphi}\left(\partial \mathcal{A}_{0}^{(23)}\right)^{2}$ in the scalar Lagrangian. In addition, there is an abelian $\mathbb{R}$ symmetry under which only the axion $A_{0}^{(123)}$ transforms, by a constant shift $A_{0}^{(123)} \rightarrow A_{0}^{(123)}+$ const. Furthermore, we find that there is another $\mathbb{R}$ symmetry, corresponding to a constant shift of the linear combination of the dilatons that is orthogonal both to $\vec{b}_{123}$ and to $\vec{b}_{23}$. Under this transformation, the various gauge potentials must scale by appropriate exponentials of the shift parameter, so as to compensate for the scalings of the dilaton prefactors in their kinetic terms. A non-trivial check on the consistency of these scalings is that the dilaton vectors associated with the mass terms for the massive fields and also the dilaton vectors associated with their kinetic terms must have common projections onto the direction orthogonal to $\vec{b}_{123}$ and $\vec{b}_{23}$. Thus, in all, we find a symmetry

$$
S L(2, \mathbb{R}) \times \mathbb{R} \times \mathbb{R}
$$

of the massive theory described by 2.8 .

\subsection{Scherk-Schwarz reduction of $D=8$ supergravity}

The Lagrangian for the bosonic sector of massless $D=8$ maximal supergravity, in the notation given in the Appendix, is given by

$$
\begin{aligned}
e^{-1} \mathcal{L}= & R-\frac{1}{2}\left(\partial \phi_{1}\right)^{2}-\frac{1}{2}\left(\partial \phi_{2}\right)^{2}-\frac{1}{2}\left(\partial \phi_{3}\right)^{2}-\frac{1}{2} e^{\vec{b}_{12} \cdot \vec{\phi}}\left(\mathcal{F}_{1}^{(12)}\right)^{2} \\
& -\frac{1}{2} e^{\vec{b}_{13} \cdot \vec{\phi}}\left(\mathcal{F}_{1}^{(13)}\right)^{2}-\frac{1}{2} e^{\vec{b}_{23} \cdot \vec{\phi}}\left(\mathcal{F}_{1}^{(23)}\right)^{2}-\frac{1}{2} e^{\vec{a}_{123} \cdot \vec{\phi}}\left(F_{1}^{(123)}\right)^{2}-\frac{1}{48} e^{\vec{a} \cdot \vec{\phi}} F_{4}^{2} \\
& -\frac{1}{12} e^{\vec{a}_{1} \cdot \vec{\phi}}\left(F_{3}^{(1)}\right)^{2}-\frac{1}{12} e^{\vec{a}_{2} \cdot \vec{\phi}}\left(F_{3}^{(2)}\right)^{2}-\frac{1}{12} e^{\vec{a}_{3} \cdot \vec{\phi}}\left(F_{3}^{(3)}\right)^{2}-\frac{1}{4} e^{\vec{a}_{12} \cdot \vec{\phi}}\left(F_{2}^{(12)}\right)^{2} \\
& -\frac{1}{4} e^{\vec{a}_{13} \cdot \vec{\phi}}\left(F_{2}^{(13)}\right)^{2}-\frac{1}{4} e^{\vec{a}_{23} \cdot \vec{\phi}}\left(F_{2}^{(23)}\right)^{2}-\frac{1}{4} e^{\vec{b}_{1} \cdot \vec{\phi}}\left(\mathcal{F}_{2}^{(1)}\right)^{2} \\
& -\frac{1}{4} e^{\vec{b}_{2} \cdot \vec{\phi}}\left(\mathcal{F}_{2}^{(2)}\right)^{2}-\frac{1}{4} e^{\vec{b}_{3} \cdot \vec{\phi}}\left(\mathcal{F}_{2}^{(3)}\right)^{2}+e^{-1} \mathcal{L}_{F F A}
\end{aligned}
$$

There are now four axions in total, namely $\mathcal{A}_{0}^{(12)}, \mathcal{A}_{0}^{(13)}, \mathcal{A}_{0}^{(23)}$ and $A_{0}^{(123)}$. The complete expressions for the Chern-Simons modifications to the field strengths, given by (A.5), are quite involved, and we shall not write them out explicitly. As we saw in the previous subsection, the basic structure of the Scherk-Schwarz reduced theory is governed by the bilinear terms in those Chern-Simons modifications that involve the axion being used for the reduction process. Thus, for the purposes of determining the spectrum of massive 
and massless fields in the Scherk-Schwarz reduced theories, it suffices to consider only the bilinear Chern-Simons modifications involving the four axions. From the general results of [11], summarised in the Appendix, we therefore have:

$$
\begin{aligned}
\mathcal{F}_{1}^{(12)} & =\tilde{\mathcal{F}}_{1}^{(12)}, \quad \mathcal{F}_{1}^{(13)}=\tilde{\mathcal{F}}_{1}^{(13)}-\mathcal{A}_{0}^{(23)} \tilde{\mathcal{F}}_{1}^{(12)}, \quad \mathcal{F}_{1}^{(23)}=\tilde{\mathcal{F}}_{1}^{(23)}, \\
\mathcal{F}_{2}^{(1)} & =\tilde{\mathcal{F}}_{2}^{(1)}+\mathcal{A}_{1}^{(2)} \wedge \tilde{\mathcal{F}}_{1}^{(12)}+\mathcal{A}_{1}^{(3)} \wedge \tilde{\mathcal{F}}_{1}^{(13)}+\cdots, \\
\mathcal{F}_{2}^{(2)} & =\tilde{\mathcal{F}}_{2}^{(2)}+\mathcal{A}_{1}^{(3)} \wedge \tilde{\mathcal{F}}_{1}^{(23)} \\
\mathcal{F}_{2}^{(3)} & =\tilde{\mathcal{F}}_{2}^{(3)}, \quad F_{1}^{(123)}=\tilde{F}_{1}^{(123)} \\
F_{2}^{(12)} & =\tilde{F}_{2}^{(12)}+\mathcal{A}_{1}^{(3)} \wedge \tilde{F}_{1}^{(123)} \\
F_{2}^{(13)} & =\tilde{F}_{2}^{(13)}-\mathcal{A}_{0}^{(23)} \tilde{F}_{2}^{(12)}-\mathcal{A}_{1}^{(2)} \wedge \tilde{F}_{1}^{(123)}, \\
F_{2}^{(23)} & =\tilde{F}_{2}^{(23)}-\mathcal{A}_{0}^{(12)} \tilde{F}_{2}^{(13)}+\mathcal{A}_{0}^{(13)} \tilde{F}_{2}^{(12)}+\mathcal{A}_{1}^{(1)} \wedge \tilde{F}_{1}^{(123)} \\
F_{3}^{(1)} & =\tilde{F}_{3}^{(1)}+\cdots, \quad F_{3}^{(2)}=\tilde{F}_{3}^{(2)}-\mathcal{A}_{0}^{(12)} \tilde{F}_{3}^{(1)}+\cdots \\
F_{3}^{(3)} & =\tilde{F}_{3}^{(3)}-\mathcal{A}_{0}^{(13)} \tilde{F}_{3}^{(1)}-\mathcal{A}_{0}^{(23)} \tilde{F}_{3}^{(2)}+\cdots \\
F_{4} & =\tilde{F}_{4}+\cdots,
\end{aligned}
$$

where the dots $\cdots$ indicate that higher-order terms, or bilinear terms that do not involve the axions, have been omitted.

Let us now consider the various massive $D=7$ supergravity theories that one can obtain by carrying out a Scherk-Schwarz reduction step using one or another of the four axions. We shall begin by considering the axion $\mathcal{A}_{0}^{(12)}$. Certain field redefinitions are required in order to recast the massless $D=8$ theory into a form in which the field $\mathcal{A}_{0}^{(12)}$ is covered by a derivative everywhere, namely

$$
A_{1}^{(23)} \longrightarrow A_{1}^{(23)}+\mathcal{A}_{0}^{(12)} A_{1}^{(13)}, \quad A_{2}^{(2)} \longrightarrow A_{2}^{(2)}+\mathcal{A}_{0}^{(12)} A_{2}^{(1)} .
$$

One can easily check that these redefinitions, whose form is dictated by requiring that $\mathcal{A}_{0}^{(12)}$ be covered by a derivative everywhere in the bilinear Chern-Simons modifications displayed in (2.12), are also sufficient to ensure that it is covered by a derivative in all of the higher-order terms too.

In a similar manner, if instead we wish to use $\mathcal{A}_{0}^{(13)}$ for the Scherk-Schwarz reduction, we may ensure that it is covered by derivatives everywhere by performing instead the following field redefinitions

$$
A_{1}^{(23)} \longrightarrow A_{1}^{(23)}-\mathcal{A}_{0}^{(13)} A_{1}^{(12)}, \quad A_{2}^{(3)} \longrightarrow A_{2}^{(3)}+\mathcal{A}_{0}^{(13)} A_{2}^{(1)}
$$

\footnotetext{
${ }^{4}$ In fact, as we shall discuss later, it is possible to cover more than one axion with derivatives simultaneously, thereby permitting more exotic kinds of Scherk-Schwarz reductions.
} 
The third of the axions of the $\mathcal{A}_{0}^{(i j)}$ type, namely $\mathcal{A}_{0}^{(23)}$, can be covered by a derivative everywhere if we instead redefine fields according to:

$$
\begin{aligned}
& \mathcal{A}_{0}^{(13)} \longrightarrow \mathcal{A}_{0}^{(13)}+\mathcal{A}_{0}^{(12)} \mathcal{A}_{0}^{(23)}, \\
& A_{1}^{(13)} \longrightarrow A_{1}^{(13)}+\mathcal{A}_{0}^{(23)} A_{1}^{(12)}, \quad A_{2}^{(3)} \longrightarrow A_{2}^{(3)}+\mathcal{A}_{0}^{(23)} A_{2}^{(2)} .
\end{aligned}
$$

Finally, we note that the fourth axion, $A_{0}^{(123)}$, already appears covered by a derivative everywhere in the Chern-Simons modifications to the field strengths. It does, however, appear undifferentiated in the $\mathcal{L}_{F F A}$ term, given in (A.8). This is easily taken care of by performing an integration by parts on the term where this field appears in the action. Thus, we have seen that we can perform a Scherk-Schwarz reduction on any of the four axions of the $D=8$ supergravity theory.

For the first three axions, $\mathcal{A}_{0}^{(i j)}$, where there are no contributions arising from the $\mathcal{L}_{F F A}$ term, the spectrum of massive fields is determined in precisely the manner described in the previous subsection. If we use $\mathcal{A}_{0}^{(12)}$ for the Scherk-Schwarz reduction, we find that three 2-form field strengths become massive, corresponding to the fields $\mathcal{A}_{1}^{(4)}, \mathcal{A}_{1}^{(2)}$ and $A_{1}^{(13)}$. It turns out that there is then a residual $S L(2, \mathbb{R})$ symmetry of the scalar manifold, under which the first of these fields is a singlet, while the latter two form a doublet. There is also a massive 3-form field strength, corresponding to $A_{2}^{(1)}$, and a massive 1-form field strength corresponding to $\mathcal{A}_{0}^{(23)}$. Both of these are singlets under $S L(2, \mathbb{R})$. The complete structure of massive and massless fields is displayed in Table 1 below. The fields enclosed in angle brackets in the massive column indicate those that are absorbed by the corresponding fields appearing directly above them. The axion $A_{0}^{(123)}$ is enclosed in square brackets, to indicate that it is the "ignorable coordinate" $\chi$, appearing always differentiated, in the $S L(2, \mathbb{R}) / S O(2)$ scalar manifold. The associated dilatonic scalar in this manifold is the combination $\varphi \equiv-\frac{1}{2} \vec{a}_{123} \cdot \vec{\phi}$. Note that $B_{2}$ is the 2 -form potential for the dual of the 4 -form field strength, i.e. ${ }^{*} F_{4}=d B_{2}$. The dilaton vector for the cosmological term $\frac{1}{2} m^{2} e^{\vec{c} \cdot \vec{\phi}}$ in the $D=7$ massive theory is given by $\vec{c}=\vec{b}_{124}$ in this case, where $\vec{b}_{124}$ is defined in (A.2). 


\begin{tabular}{|c|c|c|}
\hline Dimension & Massive & Massless \\
\hline \hline Doublets & $\left(\mathcal{A}_{1}^{(2)}, A_{1}^{(13)}\right)$ & $\left(A_{2}^{(4)}, B_{2}\right)$ \\
& $\left\langle\mathcal{A}_{0}^{(14)}, A_{0}^{(234)}\right\rangle$ & $\left(\mathcal{A}_{1}^{(3)}, A_{1}^{(12)}\right)$ \\
& & $\left(\mathcal{A}_{0}^{(24)}, A_{0}^{(134)}\right)$ \\
& & $\left(\mathcal{A}_{0}^{(34)}, A_{0}^{(124)}\right)$ \\
\hline Singlets & $A_{2}^{(1)}$ & $A_{2}^{(2)} A_{2}^{(3)}$ \\
& $\left\langle A_{1}^{(24)}\right\rangle$ & $\mathcal{A}_{1}^{(1)} A_{1}^{(14)} A_{1}^{(23)} A_{1}^{(34)}$ \\
& $\mathcal{A}_{1}^{(4)}$ & $\mathcal{A}_{0}^{(13)}\left[A_{0}^{(123)}\right]$ \\
& $\left\langle\mathcal{A}_{0}^{(12)}\right\rangle$ & \\
& $\mathcal{A}_{0}^{(23)}$ & \\
\hline
\end{tabular}

Table 1: $S L(2, \mathbb{R})$ multiplets for the $\mathcal{A}_{0}^{(12)}$ reduction

If the axion $\mathcal{A}_{0}^{(13)}$ is used instead for the Scherk-Schwarz reduction, then we find that the fields $\left(\mathcal{A}_{1}^{(4)}, A_{1}^{(12)}, \mathcal{A}_{1}^{(3)}\right)$ become massive, absorbing $\left\langle\mathcal{A}_{0}^{(13)}, A_{0}^{(234)}, \mathcal{A}_{0}^{(14)}\right\rangle$ in the process. We find that these fields form triplets under a residual $S L(3, \mathbb{R})$ symmetry of the scalar manifold. The $S L(3, \mathbb{R}) / S O(3)$ manifold has three axionic ignorable coordinates $\left[\mathcal{A}_{0}^{(34)}, A_{0}^{(123)}, A_{0}^{(124)}\right]$, Their associated dilatonic scalars are the two combinations $\varphi_{1}=$ $-\frac{1}{2} \vec{b}_{34} \cdot \vec{\phi}$ and $\varphi_{2}=-\frac{1}{2 \sqrt{3}}\left(\vec{a}_{123}+\vec{a}_{124}\right) \cdot \vec{\phi}$. There is also a singlet massive field $A_{2}^{(1)}$, which absorbs $A_{1}^{(34)}$. These, and the remaining massless fields, are listed Table 2 below. They also fall into triplets and singlets under the $S L(3, \mathbb{R})$ symmetry group. As above, in the massive column, the fields enclosed in angle brackets are absorbed by those immediately above them. The dilaton vector for the cosmological term is given by $\vec{c}=\vec{b}_{134}$ in this case.

\begin{tabular}{|c|c|c|}
\hline Dimension & Massive & Massless \\
\hline \hline Triplets & $\left(\mathcal{A}_{1}^{(4)}, A_{1}^{(12)}, \mathcal{A}_{1}^{(3)}\right)$ & $\left(A_{2}^{(3)}, A_{2}^{(4)}, B_{2}\right)$ \\
& $\left\langle\mathcal{A}_{0}^{(13)}, A_{0}^{(234)}, \mathcal{A}_{0}^{(14)}\right\rangle$ & $\left(\mathcal{A}_{1}^{(1)}, A_{1}^{(23)}, A_{1}^{(24)}\right)$ \\
& & $\left(\mathcal{A}_{1}^{(2)}, A_{1}^{(13)}, A_{1}^{(14)}\right)$ \\
& & $\left(\mathcal{A}_{0}^{(23)}, \mathcal{A}_{0}^{(24)}, A_{0}^{(134)}\right)$ \\
& & {$\left[\mathcal{A}_{0}^{(34)}, A_{0}^{(123)}, A_{0}^{(124)}\right]$} \\
\hline Singlets & & $A_{2}^{(2)}$ \\
& $A_{2}^{(1)}$ & $\mathcal{A}_{0}^{(12)}$ \\
\hline
\end{tabular}

Table 2: $S L(3, \mathbb{R})$ multiplets for the $\mathcal{A}_{0}^{(13)}$ reduction 
Turning now to the Scherk-Schwarz reduction using the third of the $\mathcal{A}_{0}^{(i j)}$ axions, namely $\mathcal{A}_{0}^{(23)}$, we find once again there is an $S L(3, \mathbb{R})$ symmetry of the scalar manifold, with $\left[\mathcal{A}_{0}^{(34)}, A_{0}^{(123)}, A_{0}^{(124)}\right]$ as the ignorable coordinates. Their associated dilatonic scalars are the two combinations $\varphi_{1}=-\frac{1}{2} \vec{b}_{23} \cdot \vec{\phi}$ and $\varphi_{2}=-\frac{1}{2 \sqrt{3}}\left(\vec{a}_{123}+\vec{a}_{124}\right) \cdot \vec{\phi}$. The triplets and singlets under $S L(3, \mathbb{R})$ are listed in Table 3 below, using the same notation as in the previous cases. The dilaton vector for the cosmological term is $\vec{c}=\vec{b}_{234}$. Note that, although the surviving $S L(3, \mathbb{R})$ symmetry group for this case is the same as for the Scherk-Schwarz reduction using $\mathcal{A}_{0}^{(13)}$, the two $D=7$ theories are quite distinct; in particular, there is a massive axion in the $\mathcal{A}_{0}^{(23)}$ reduction, while in the $\mathcal{A}_{0}^{(13)}$ reduction the analogous axion is massless.

\begin{tabular}{|c|c|c|}
\hline Dimension & Massive & Massless \\
\hline \hline Triplets & $\left(\mathcal{A}_{1}^{(3)}, \mathcal{A}_{1}^{(4)}, A_{1}^{(12)}\right)$ & $\left(A_{2}^{(3)}, A_{2}^{(4)}, B_{2}\right)$ \\
& $\left\langle\mathcal{A}_{0}^{(23)}, \mathcal{A}_{0}^{(24)}, A_{0}^{(134)}\right\rangle$ & $\left(\mathcal{A}_{1}^{(1)}, A_{1}^{(23)}, A_{1}^{(24)}\right)$ \\
& & $\left(\mathcal{A}_{1}^{(2)}, A_{1}^{(13)}, A_{1}^{(14)}\right)$ \\
& & $\left(\mathcal{A}_{0}^{(13)}, \mathcal{A}_{0}^{(14)}, A_{0}^{(234)}\right)$ \\
& & {$\left[\mathcal{A}_{0}^{(34)}, A_{0}^{(123)}, A_{0}^{(124)}\right]$} \\
\hline Singlets & & \\
& & $A_{2}^{(1)}$ \\
& $\left\langle A_{1}^{(34)}\right\rangle$ & \\
& $\mathcal{A}_{0}^{(12)}$ & \\
\hline
\end{tabular}

Table 3: $S L(3, \mathbb{R})$ multiplets for the $\mathcal{A}_{0}^{(23)}$ reduction

Finally, we turn to the Scherk-Schwarz reduction using the axion $A_{0}^{(123)}$ derived from the 3 -form potential occurring in $D=11$. In this case, we find that there is a residual $S L(4, \mathbb{R})$ symmetry of the scalar manifold, with all of the axions $\left[\mathcal{A}_{0}^{(i j)}\right]$ being ignorable coordinates. The associated dilatonic coordinates are the three combinations of the $\vec{\phi}$ fields that are orthogonal to the dilaton vector for the cosmological term, which is given by $\vec{c}=\vec{a}_{1234}$ in this case. The various fields fall into sextets, quartets and singlets under $S L(4, \mathbb{R})$, as indicated in Table 4 below. Note that, in this case, the field $A_{3}$ becomes massive, not by the usual mechanism of absorbing a 2-form potential, but instead by acquiring a topological mass term in the $D=7$ Lagrangian. This arises because the term $-\frac{1}{2} \tilde{F}_{4} \wedge \tilde{F}_{4} A_{0}^{(123)}$ in the $D=8 \mathcal{L}_{F F A}$ term gives $-\frac{1}{2} \tilde{F}_{4} \wedge A_{3} \wedge d A_{0}^{(123)}$ after integration by parts, and this then reduces, on imposing the Scherk-Schwarz reduction ansatz $A_{0}^{(123)}(x, z) \rightarrow m z+A_{0}^{(123)}(x)$, 
to give a term

$$
-\frac{1}{2} m F_{4} \wedge A_{3}
$$

in the $D=7$ Lagrangian. The supergravity theory arising in this case is the maximal $N=2$ extension of the $N=1$ theory with a topological mass term found in 16] (with the Yang-Mills gauge coupling constant set equal to zero).

\begin{tabular}{|c|c|c|}
\hline Dimension & Massive & Massless \\
\hline \hline Sextets & & $\left(A_{1}^{(12)}, A_{1}^{(13)}, A_{1}^{(14)}, A_{1}^{(23)}, A_{1}^{(24)}, A_{1}^{(34)}\right)$ \\
& & $\begin{array}{c}(12) \\
{\left[\mathcal{A}_{0}^{(12)}, \mathcal{A}_{0}^{(13)}, \mathcal{A}_{0}^{(14)}, \mathcal{A}_{0}^{(23)}, \mathcal{A}_{0}^{(24)}, A_{0}^{(34)}\right]}\end{array}$ \\
\hline Quadruplets & $\left(\mathcal{A}_{1}^{(1)}, \mathcal{A}_{1}^{(2)}, \mathcal{A}_{1}^{(3)}, \mathcal{A}_{1}^{(4)}\right)$ & $\left(A_{2}^{(1)}, A_{2}^{(2)}, A_{2}^{(3)}, A_{2}^{(4)}\right)$ \\
& $\left\langle A_{0}^{(234)}, A_{0}^{(134)}, A_{0}^{(124)}, A_{0}^{(123)}\right\rangle$ & \\
\hline Singlets & $A_{3}$ & \\
\hline
\end{tabular}

Table 4: $S L(4, \mathbb{R})$ multiplets for the $A_{0}^{(123)}$ reduction

We have seen above that, depending on the choice of which of the four axions of the $D=8$ theory is used for the Scherk-Schwarz reduction step, we can obtain four inequivalent massive $D=7$ maximal supergravity theories. It is also easy to see that we get two further inequivalent theories arising in $D=7$ by performing ordinary Kaluza-Klein reductions of the massive IIA theory in $\mathrm{D}=10$, and of the massive theory in $D=8$ that was discussed in the previous subsection. The simplest way of seeing the inequivalence of these is by observing that the massive IIA theory, for which just a single 2-form potential is massive in $D=10$, will give a theory in $D=7$ that has one massive 2 -form potential, three massive 1 -forms, and three massive axions in $D=7$. On the other hand, the massive $D=8$ theory constructed in the previous subsection has a massive 2 -form potential and two massive 1 -forms in $D=8$, yielding, after ordinary Kaluza-Klein reduction, a massive 2-form potential, three massive 1-forms, and two massive axions in $D=7$. These two theories thus differ in their axionic sectors from the four already-inequivalent theories constructed above. Although these latter have the same counting of massive 2-form and 1-form potentials, they have either no massive axion, or one massive axion.

It is of interest also to examine the surviving symmetries of the scalar Lagrangians in $D=7$ for the two additional theories just discussed, obtained either from the dimensional reduction of the massive IIA theory in $D=10$, or from the new massive theory in $D=8$ that we obtained in the previous subsection. We shall not present the details here, but simply record that the first of these two theories has a surviving $S L(3, \mathbb{R})$ symmetry in 
$D=7$, while the second has an $S L(2, \mathbb{R})$ symmetry. Finally, we remark that, as in the case of the massive $D=8$ supergravity constructed in the previous subsection, one can also seek in all six of the $D=7$ theories for additional abelian symmetries, associated either with shift symmetries of the axions that do not participate as ignorable coordinates in the non-abelian coset manifolds as already considered, or else associated with shift symmetries of dilatonic scalars, together with appropriate rescalings of the gauge potentials. Abelian symmetries of the former type are somewhat involved to analyse in general, since one needs to determine how many of the "surplus" axions admit shift symmetries (related issues are discussed in the next section). Extra abelian symmetries of the second type are easier to classify. We find that each combination of dilatonic scalars that is orthogonal to the dilaton vector for the cosmological term, and that is also orthogonal to all of the dilaton vectors for the axions involved in the non-abelian part of the scalar manifold, allows such an independent abelian symmetry. This implies that the non-abelian symmetries $S L(2, \mathbb{R})$, $S L(3, \mathbb{R})$ and $S L(4, \mathbb{R})$ which we found for the various $D=7$ theories become (at least) $S L(2, \mathbb{R}) \times \mathbb{R} \times \mathbb{R}, S L(3, \mathbb{R}) \times \mathbb{R}$, and $S L(4, \mathbb{R})$ respectively, when the dilatonic shift symmetries are included.

As we proceed to lower dimensions, the numbers of inequivalent massive theories proliferates. The numbers of axions in $D=7,6,5$ and 4 are 10, 20, 36 and 63 respectively. In each dimension, each axion, if used for a Scherk-Schwarz reduction of the kind we are considering here, will give rise to a different massive theory in one lower dimension. Furthermore, we accumulate additional inequivalent theories from the ordinary Kaluza-Klein reductions of higher-dimensional massive theories. Thus, the total numbers of massive theories of the kind we are constructing here will be 15, 35, 71 and 134 in $D=6,5,4$ and 3 , respectively. In addition, there is one more massive theory in each dimension that comes from the Kaluza-Klein reduction of the massive IIA theory in $D=10$. The massive theories may admit consistent truncations to theories with less than maximal supersymmetry. For example in $D=4$, some of the 72 massive $N=8$ theories may admit truncations to $N=1$, in which case they may reduce to the models considered in [17], for which domain-wall solutions were given.

\footnotetext{
${ }^{5}$ In $D=5$ we are including $A_{3}$ in the counting of axions, since its field strength $F_{4}$ can be dualised to a 1-form field strength for another axion. Similarly, in $D=4$ we are including the 2-form potentials $A_{2}^{(i)}$. As we shall discuss further in the conclusions, for the purposes of constructing consistent truncations of $D=11$ to give lower-dimensional theories, it is better to leave these fields in their original undualised formulations, in which case they would reduce by the ordinary Kaluza-Klein procedure rather than ScherkSchwarz procedure.
} 


\section{Scherk-Schwarz reduction using multiple axions}

In the examples of Scherk-Schwarz reduction that we have considered so far, we have imposed the standard Kaluza-Klein ansatz on all of the higher-dimensional fields, with the exception of a single axion, for which the more general ansatz (2.7) was allowed. As we discussed above, the ability to perform such a consistent truncation depends upon the fact that there exists a choice of field variables in which the chosen axion $\chi$ is covered by a derivative everywhere in the the higher-dimensional Lagrangian. Although we did not exploit it in the previous section, we also remarked that one can in fact typically choose field variables in the higher dimension such that more than one of the axions are simultaneously covered by derivatives everywhere. Under these circumstances, it is then possible to perform a family of Scherk-Schwarz reductions in which all the derivative-covered axions satisfy ansätze of the form (2.7), with independent mass parameters for each. We shall explore below the structure of the resulting lower-dimensional theories that are obtained in such cases. The counting of the number of axions that can simultaneously be covered with derivatives is a somewhat involved one in general, and we shall not attempt a complete discussion. Clearly in principle it can be established, dimension by dimension, by enumerating all of the cases. Rather than do this, we shall make some general observations, and then shall consider the example of $D=8$ supergravity in detail, to illustrate the possibilities.

From the general form of the Lagrangian for maximal supergravity in $D$ dimensions, as given in the Appendix, it is clear that all of the axions of the form $A_{0}^{(i j k)}$ can easily be simultaneously covered by derivatives, without the need for any field redefinitions. They already appear in the desired form in the Chern-Simons modifications (A.5), while in the $\mathcal{L}_{F F A}$ terms (A.8) it is a simple matter of performing appropriate integrations by parts in order to cover them with derivatives there too. In addition, it is possible that appropriate field redefinitions can allow one or more of the $\mathcal{A}_{0}^{(i j)}$ axions to be simultaneously covered as well. The enumeration of all the possibilities is complicated, however, since it may well be,

for example, that more axions of the $\mathcal{A}_{0}^{(i j)}$ type can be covered if fewer of the $A_{0}^{(i j k)}$ axions are covered, and so on.

Let us now consider the specific example of $D=8$ supergravity, which contains four axions; $\mathcal{A}_{0}^{(12)}, \mathcal{A}_{0}^{(13)}, \mathcal{A}_{0}^{(23)}$ and $A_{0}^{(123)}$. It is clear from the expression for $\mathcal{F}_{1}^{(13)}$ in 2.12) that it is not possible to put derivatives on $\mathcal{A}_{0}^{(12)}$ and $\mathcal{A}_{0}^{(23)}$ simultaneously. We can, however, cover three out of the four axions, for example $\mathcal{A}_{0}^{(12)}, \mathcal{A}_{0}^{(13)}$ and $A_{0}^{(123)}$. This is achieved by 
making the field redefinitions

$$
\begin{aligned}
A_{1}^{(23)} & \longrightarrow A_{1}^{(23)}-\mathcal{A}_{0}^{(13)} A_{1}^{(12)}+\mathcal{A}_{0}^{(12)} A_{1}^{(13)} \\
A_{2}^{(2)} & \longrightarrow A_{2}^{(2)}+\mathcal{A}_{0}^{(12)} A_{2}^{(1)}, \quad A_{2}^{(3)} \longrightarrow A_{2}^{(3)}+\mathcal{A}_{0}^{(13)} A_{2}^{(1)}
\end{aligned}
$$

together with the necessary intergration by parts in the $\mathcal{L}_{F F A}$ term.

We shall now discuss the general structure of the $D=7$ theory that results from ScherkSchwarz reduction of the $D=8$ theory, after the redefinitions (3.1), with the ansätze

$$
\begin{aligned}
& \mathcal{A}_{0}^{(12)}(x, z) \quad m_{1} z+\mathcal{A}_{0}^{(12)}(x) \\
& \mathcal{A}_{0}^{(13)}(x, z) \quad \longrightarrow m_{2} z+\mathcal{A}_{0}^{(13)}(x) \\
& A_{0}^{(123)}(x, z) \quad \longrightarrow m_{3} z+A_{0}^{(123)}(x)
\end{aligned}
$$

We find that the form of the resulting theory in $D=7$ is

$$
\begin{aligned}
e^{-1} \mathcal{L}= & R-\frac{1}{2}(\partial \vec{\phi})^{2}-\frac{1}{2} e^{\vec{b}_{12} \cdot \vec{\phi}}\left(\partial \mathcal{A}_{0}^{(12)}-m_{1} \mathcal{A}_{1}^{(4)}\right)^{2} \\
& -\frac{1}{2} e^{\vec{b}_{13} \cdot \vec{\phi}}\left(\partial \mathcal{A}_{0}^{(13)}-m_{2} \mathcal{A}_{1}^{(4)}-\mathcal{A}_{0}^{(23)} \partial \mathcal{A}_{0}^{(12)}+m_{1} \mathcal{A}_{0}^{(23)} \mathcal{A}_{1}^{(4)}\right)^{2} \\
& -\frac{1}{2} e^{\vec{b}_{14} \cdot \vec{\phi}}\left(\partial \mathcal{A}_{0}^{(14)}+m_{1} \mathcal{A}_{1}^{(2)}+m_{2} \mathcal{A}_{1}^{(3)}\right)^{2}-\frac{1}{2} e^{\vec{b}_{23} \cdot \vec{\phi}}\left(\partial \mathcal{A}_{0}^{(23)}\right)^{2}-\frac{1}{2} e^{\vec{b}_{24} \cdot \vec{\phi}}\left(\partial \mathcal{A}_{0}^{(24)}\right)^{2} \\
& -\frac{1}{2} e^{\vec{b}_{34} \cdot \vec{\phi}}\left(\partial \mathcal{A}_{0}^{(34)}\right)^{2}-\frac{1}{2} e^{\vec{a}_{123} \cdot \vec{\phi}}\left(\partial A_{0}^{(123)}-m_{3} \mathcal{A}_{1}^{(4)}\right)^{2} \\
& -\frac{1}{2} e^{\vec{a}_{124} \cdot \vec{\phi}}\left(\partial A_{0}^{(124)}+m_{3} \mathcal{A}_{1}^{(3)}\right)^{2}-\frac{1}{2} e^{\vec{a}_{134} \cdot \vec{\phi}}\left(\partial A_{0}^{(134)}-m_{3} \mathcal{A}_{1}^{(2)}\right)^{2} \\
& -\frac{1}{2} e^{\vec{a}_{234} \cdot \vec{\phi}}\left(\partial A_{0}^{(234)}+m_{3} \mathcal{A}_{1}^{(1)}+m_{1} A_{1}^{(13)}-m_{2} A_{1}^{(12)}\right)^{2}-\frac{1}{4} \sum_{i=1}^{4} e^{\vec{b}_{i} \cdot \vec{\phi}}\left(\mathcal{F}_{2}^{(i)}\right)^{2} \\
& -\frac{1}{4} \sum_{i<j \leq 3} e^{\vec{a}_{i j} \cdot \vec{\phi}}\left(F_{2}^{(i j)}\right)^{2}-\frac{1}{4} e^{\vec{a}_{14} \cdot \vec{\phi}}\left(F_{2}^{(14)}\right)^{2}-\frac{1}{4} e^{\vec{a}_{24} \cdot \vec{\phi}}\left(F_{2}^{(24)}+m_{1} A_{2}^{(1)}\right)^{2} \\
& -\frac{1}{4} e^{\vec{a}_{34} \cdot \vec{\phi}}\left(F_{2}^{(34)}+m_{2} A_{2}^{(1)}\right)^{2}-\frac{1}{12} \sum_{i=1}^{4} e^{\vec{a}_{i} \cdot \vec{\phi}}\left(F_{3}^{(i)}\right)^{2}-\frac{1}{48} e^{\vec{a} \cdot \vec{\phi}} F_{4}^{2}-\frac{1}{2} m_{3} F_{4} \wedge A_{3} \\
& -\frac{1}{2} m_{1}^{2} e^{\vec{b}_{124} \cdot \vec{\phi}}-\frac{1}{2} m_{3}^{2} e^{\vec{a}_{1234} \cdot \vec{\phi}}-\frac{1}{2}\left(m_{2}-m_{1} \mathcal{A}_{0}^{(23)}\right)^{2} e^{\vec{b}_{134} \cdot \vec{\phi}}+\cdots
\end{aligned}
$$

where we have suppressed certain higher-order terms that are not of relevance for the present discussion.

We see from the final line of the Lagrangian that there are two standard-type cosmological terms, namely those with coefficients $m_{1}^{2}$ and $m_{3}^{2}$, together with the final term, which requires some further discussion. If $m_{1}$ is non-zero, we can make the field redefinition

$$
\mathcal{A}_{0}^{(23)} \longrightarrow \mathcal{A}_{0}^{(23)}+\frac{m_{2}}{m_{1}}
$$

in which case the final term becomes a standard mass term for $\mathcal{A}_{0}^{(23)}$. Note also that, at the same time, the $m_{2} \mathcal{A}_{1}^{(4)}$ term in the kinetic term $\left(\partial \mathcal{A}_{0}^{(13)}+\cdots\right)^{2}$ is removed. On the 
other hand, if $m_{1}=0$, then the final term in the Lagrangian (3.3) becomes a standard cosmological term, while the first cosmological term, $-\frac{1}{2} m_{1}^{2} e^{\vec{b}_{124} \cdot \vec{\phi}}$, disappears. Thus, either way, there are two cosmological-type terms in the $D=7$ Lagrangian.

In lower dimensions, the possibilities for performing simultaneous Scherk-Schwarz reductions on multiple axions become more numerous. For example, we have found by explicit computation that in all dimensions, all the axions of the form $\mathcal{A}_{0}^{(1 a)}$ and $A_{0}^{(a b c)}$ can simultaneously be covered by derivatives, where the indices $a, b, \ldots$ run over the values 2 to $11-D$. It is interesting that these are precisely the Ramond-Ramond axions, from the viewpoint of the type IIA string [1]. In $D=5$, there is another field that describes an axionic-type degree of freedom, namely $A_{3}$, whose field strength $F_{4}$ is dual to another 1-form field strength. The field $A_{3}$ is in the Ramond-Ramond sector, and we find that in $D=5$ it too can be covered by derivatives at the same time as the $\mathrm{RR}$ axions $\mathcal{A}_{0}^{(1 a)}$ and $A_{0}^{(a b c)}$. Similarly, in $D=4$ where the potentials $A_{2}^{(i)}$ describe axionic-type degrees of freedom, we find that the Ramond-Ramond subset $A_{2}^{(a)}$ can be covered by derivatives at the same time as the RR axions $\mathcal{A}_{0}^{(1 a)}$ and $A_{0}^{(a b c)}$. Thus in total we have $2^{9-D}$ RR fields in $D$ dimensions that can be simultaneously covered by derivatives.

Other sets of axions may also be covered by derivatives simultaneously. For example, we have already seen in this section that in $D=8$, we may cover three of the four axions simultaneously. These comprise the two Ramond-Ramond axions $\mathcal{A}_{0}^{(12)}$ and $\mathcal{A}_{0}^{(12)}$, together with the NS-NS axion $A_{0}^{(123)}$. Another example, in $D=4$, is the set of all 35 axions $A_{0}^{(i j k)}$, which can be covered by derivatives simultaneously. These comprise 20 Ramond-Ramond fields $A_{0}^{(a b c)}$ and 15 NS-NS fields $A_{0}^{(1 a b)}$, and their total number exceeds the total of 32 Ramond-Ramond axions described above. Using all the $A_{0}^{(i j k)}$ axions, a Scherk-Schwarz reduction to $D=3$ can be performed that involves 35 independent mass parameters. However, this does not imply that there will be 35 different cosmological terms in $D=3$, just as we saw above in $D=7$ that there are 2 cosmological terms rather than 3 . In $D=7$, the reason for this can be identified by looking at the final term in (3.3); this indeed would be a cosmological term were it not for the appearance of the $\mathcal{A}_{0}^{(23)}$ field there, as we have seen. The presence of this term can be traced back to the Chern-Simons modification structure for the $\mathcal{F}_{1}^{(13)}$ field strength in (2.12). In fact, Chern-Simons modifications of this type are responsible for another feature in supergravity theories; namely that they govern the structure of the allowed combinations of 1-form field strengths that can participate simultaneously in $(D-3)$-brane solutions to the massless theories. The maximum number $N_{c}$ of cosmological terms in massive $D$-dimensional supergravity is the same as the maximum 
number of 1-forms that can participate in " $(D-3)$-brane" solutions in $(D+1)$ dimensions, and thus from results obtained, for example, in [11], we find that $N_{c}=1$ in $D=8 ; N_{c}=2$ in $D=7$ and $D=6 ; N_{c}=4$ in $D=5$ and $D=4$; and $N_{c}=7$ in $D=3$.

\section{Domain wall solutions}

In this section, we turn to a consideration of the $(D-2)$-brane solutions that arise in massive supergravity theories. As we discussed in the introduction, these are the natural endpoints of vertical dimensional reduction, and, in fact, we have seen that they require that the usual Kaluza-Klein reduction procedure be generalised to the Scherk-Schwarz reduction. In the dimensional reduction of the higher-dimensional supergravity theory, the Scherk-Schwarz reduction is responsible for generating the cosmological-type term occurring in the lowerdimensional Lagrangian that supports the $(D-2)$-brane solution. Thus the general form of the relevant part of the lower-dimensional Lagrangian is 12

$$
\mathcal{L}=e R-\frac{1}{2} e(\partial \phi)^{2}-\frac{1}{2} m^{2} e e^{a \phi}
$$

It is useful [5] to re-express the parameter $a$ describing the dilaton coupling in terms of the quantity $\Delta$, defined by

$$
a^{2}=\Delta+\frac{2(D-1)}{(D-2)}
$$

where $D$ denotes the spacetime dimension of the lower-dimensional theory. The equations of motion following from the Lagrangian (4.1) admit $(D-2)$-brane solutions of the form [5, 12]

$$
\begin{aligned}
d s^{2} & =H^{\frac{4}{\Delta(D-2)}} \eta_{\mu \nu} d x^{\mu} d x^{\nu}+H^{\frac{4(D-1)}{\Delta(D-2)}} d y^{2} \\
e^{\phi} & =H^{2 a / \Delta}
\end{aligned}
$$

where $H$ is an harmonic function on the 1-dimensional transverse space with coordinate $y$, of the general form $H=c \pm M y$, where $c$ is an arbitrary constant, and $M=\frac{1}{2} m \sqrt{\Delta}$. The curvature of the metric tends to zero at large values of $|y|$; it diverges if $H$ tends to zero, which can be avoided by taking $H$ to be of the form $H=c+M|y|$, with the constant $c$ being positive. There is then just a delta-function singularity in the curvature at $y=0$, corresponding to a discontinuity in the gradient of the "confining potential." The solution so obtained describes a domain wall. (The solution (4.3) in $D=4$ was also obtained in 13. For a recent review of domain-walls in $D=4$ supergravities, see [14.) 
The simplest solutions of this kind occurring in the massive supergravity theories we have constructed in this paper arise when just a single axion is used in the Scherk-Schwarz reduction procedure. In such a case, the field $\phi$ in (4.1) is just the linear combination of all the dilatonic fields $\vec{\phi}$ that lies in the direction parallel to the dilaton vector $\vec{c}$ of the cosmological term $-\frac{1}{2} m^{2} e^{\vec{c} \cdot \vec{\phi}}$ in the Scherk-Schwarz reduced theory. It is easily verified that all the possible dilaton vectors, given by $\vec{a}_{i j k \ell}$ or $\vec{b}_{i j k}$ in (A.2), have the property that they have magnitude $\sqrt{2(3 D-5) /(D-2)}$ and hence, after properly normalising $\phi$ so that it has a canonical kinetic term, we find that the value of the dilaton coupling constant $a$ in (4.1) corresponds to $\Delta=4$. Thus, in all these cases, the form of the harmonic function $H$ is $H=c \pm m y$, where $m$ is the same as the mass parameter used in the Scherk-Schwarz ansatz (2.7).

As we have discussed in the previous section, it is possible to obtain massive supergravity theories in $D \leq 7$ that have two or more cosmological terms with independent mass parameters, by performing a more general form of Scherk-Schwarz reduction using more than one axion. Just as this can be done for the $p$-brane solutions supported by charges carried by field strengths of rank $>0$, one can make a consistent truncation of such a theory with multiple cosmological terms, thereby arriving at a Lagrangian that is again of the form (4.1), but with a value of the dilaton coupling $a$ that no longer corresponds to $\Delta=4$. In fact, it is not hard to see that if the massive supergravity theory has $N_{c}$ cosmological terms, then consistent truncations to the form (4.1) with $\Delta=4 / N$ are possible, where $1 \leq N \leq N_{c}$. For example, in $D=7$ we obtain the massive theory described by (3.3), with two cosmological terms. As well as the $\Delta=4$ domain-wall solutions obtainable by using either one or the other of these, we can also construct a $\Delta=2$ domain-wall solution. The necessary truncation to a single scalar $\phi$ is achieved, as in [11], by introducing a constant unit vector $\vec{n}$, defining $\vec{\phi}=\vec{n} \phi+\vec{\phi}_{\perp}$ where $\vec{n} \cdot \vec{\phi}_{\perp}=0$, and requiring that the truncation $\vec{\phi}_{\perp}=0$ be consistent with the equations of motion for $\vec{\phi}_{\perp}$. The resulting linear algebra implies, for a truncation where the masses $m_{1}$ and $m_{3}$ are both non-zero, that $m_{1}^{2} \vec{b}_{124}+m_{3}^{2} \vec{a}_{1234}=a \vec{n}\left(m_{1}^{2}+m_{3}^{2}\right)$, and hence $m_{1}^{2}=m_{3}^{2}$ and $a^{2}=\frac{22}{5}$. From (4.2), it therefore follows that $\Delta=2$.

It is straightforward to see that the $\Delta=2$ domain wall solution in $D=7$ that we have just constructed can itself be obtained from the appropriate 5 -brane solution with $\Delta=2$ in $D=8$, by vertical dimensional reduction. This is just like the procedure for vertical reduction that we described in the introduction, where now the field strength in $D=8$ that supports the 5 -brane solution is obtained by setting equal $\mathcal{F}_{1}^{(12)}$ and $F_{1}^{(123)}$, together with 
the corresponding truncation of dilatonic scalars. In a similar manner, we may construct domain wall solutions with $\Delta=4 / N$ in any lower dimension too, for any integer $N \leq N_{c}$, where $N_{c}$ is the number of independent cosmological terms in that dimension. Once again, these solutions can all be interpreted as the vertical reductions of $(D-3)$-branes in one higher dimension, with the same values of $\Delta .^{6}$ These domain walls will preserve the same fraction of the supersymmetry as their $(D-3)$-brane oxidations, since dimensional reduction of the kind we are considering in this paper, whether of the ordinary Kaluza-Klein sort or of the more general Scherk-Schwarz sort, preserves all of the unbroken components of supersymmetry in the corresponding supergravity theories. Thus, solutions with $N=1$, $N=2$ and $N=3$ preserve $2^{-N}$ of the supersymmetry, while solutions with $N \geq 4$ all preserve $1 / 16$ of the supersymmetry [11].

We may also construct more general kinds of domain-wall solutions, precisely analogous to those that can be built for other kinds of $p$-branes. One such class of solution is the multi-scalar generalisations of the solutions with $\Delta=4 / N$ with $N \geq 2$. Such solutions exist for $p$-branes of arbitrary dimension [19], and the domain walls that we are considering in this paper are no exception. They arise by relaxing the constraint in the single-scalar solutions that the charges carried by all participating field strengths be equal. The greater generality of these solutions is achieved by having more than one independent combination of the dilatonic scalars be non-vanishing. In the context of domain-wall solutions, the independence of the charges translates into an independence of the mass parameters appearing as coefficients of the cosmological terms in the corresponding massive supergravity theories. For example, we may construct a two-scalar generalisation of the $\Delta=2$ domain wall in $D=7$ discussed above, in which the parameters $m_{1}$ and $m_{3}$ are no longer equal. In general, for a massive supergravity with cosmological terms of the form

$$
\mathcal{L}_{\text {cosmo }}=-\frac{1}{2} \sum_{\alpha=1}^{N} m_{\alpha}^{2} e^{\vec{c}_{\alpha} \cdot \vec{\phi}},
$$

multi-scalar solutions exist if the dilaton vectors satisfy $\vec{c}_{\alpha} \cdot \vec{c}_{\beta}=4 \delta_{\alpha \beta}+2(D-1) /(D-2)$; this is precisely the relation that holds for the $\Delta=4 / N$ single-scalar solutions that we discussed previously. Their multi-scalar generalisations take the form 19]

$$
d s^{2}=\prod_{\alpha=1}^{N} H_{\alpha}^{1 /(D-2)} \eta_{\mu \nu} d x^{\mu} d x^{\nu}+\prod_{\alpha=1}^{N} H_{\alpha}^{(D-1) /(D-2)} d y^{2}
$$

\footnotetext{
${ }^{6}$ There also exist more complicated $(D-3)$-brane solutions with $\Delta=24 /(N(N+1)(N+2))$, which arise as solutions of the $S L(N+1, \mathbb{R})$ Toda equations [18]. These are non-supersymmetric, and we shall not discuss their possible vertical reduction to domain walls here.
} 


$$
e^{\frac{1}{2} \varphi_{\alpha}}=H_{\alpha} \prod_{\beta=1}^{N} H_{\beta}^{(D-1) /(D-2)}
$$

where $\varphi_{\alpha} \equiv \vec{c}_{\alpha} \cdot \vec{\phi}$, and $H_{\alpha}=1+m_{\alpha}|y|$. (We have chosen the constant terms for all the harmonic functions to equal unity, for simplicity. More generally, we could allow arbitrary constants for each harmonic function.) These multi-scalar domain walls reduce to singlescalar solutions with $\Delta=4 / N$ if the mass parameters $m_{\alpha}$ are all set to be equal.

Another generalisation of the domain-wall solutions that can immediately be made is to consider the case of non-extremal domain walls. It was shown in 20 that there is a universal prescription, for $p$-brane solutions of all dimensions, for constructing non-extremal black $p$ branes from the corresponding extremal ones. When this is applied to the case of domain walls, the general multi-scalar black solutions are given by

$$
\begin{aligned}
d s^{2} & =\prod_{\alpha=1}^{N} H_{\alpha}^{1 /(D-2)}\left(-e^{2 f} d t^{2}+d x^{i} d x^{i}\right)+e^{-2 f} \prod_{\alpha=1}^{N} H_{\alpha}^{(D-1) /(D-2)} d y^{2}, \\
e^{\frac{1}{2} \varphi_{\alpha}} & =H_{\alpha} \prod_{\beta=1}^{N} H_{\beta}^{(D-1) /(D-2)},
\end{aligned}
$$

where the harmonic functions are now given by

$$
H_{\alpha}=1+k|y| \sinh ^{2} \mu_{\alpha}
$$

and the function $f$ is given by

$$
e^{2 f}=1-k|y|
$$

The previous extremal solutions are recovered in the appropriate limit, where the parameter $k$ is sent to zero at the same time as one sends the parameters $\mu_{\alpha}$ to infinity. The singlescalar black solutions are recovered if, instead, the parameters $\mu_{a}$ are set to be equal.

\section{Domain walls as higher-dimensional solutions}

We saw in the previous section that there is a correspondence between the structure of cosmological terms in a massive supergravity in $(D-1)$ dimensions obtained by Scherk-Schwarz reduction from $D$ dimensions, and the structures of the possible consistent truncations in the 1-form field strength sector of the massless supergravity in $D$ dimensions. This correspondence in fact brings us back to the discussion given in the Introduction that motivated this work. This correspondence arises precisely because any $(D-3)$-brane solution of massless supergravity in $D$ dimensions can be vertically reduced to a domain-wall solution in 
$(D-1)$ dimensions with, as we saw in the Introduction, a field configuration that forces us into a Scherk-Schwarz reduction scheme. Conversely, any domain-wall solution to one of the massive supergravities can be oxidised back to its progenitor solution in the massless supergravity whence the massive theory came.

When we make such an oxidation, we are taking advantage of the fact that all the dimensional-reduction schemes considered in this paper involve consistent truncations. Thus solutions to lower-dimensional theories may simply be reinterpreted as solutions to the higher-dimensional theories whence the lower-dimensional theories arise by reduction. From a domain-wall solution, one thus obtains by oxidation a stack of " $(D-3)$-branes" in $(D+1)$ dimensions, i.e. a solution with the same worldvolume dimension as the starting domain wall, but with an additional transverse dimension. Note that this oxidation process does not reverse the stacking-up procedure itself and does not therefore extract a single isotropic $(D-3)$-brane from the stack. To do so would change the form of the harmonic function $H(y)$, and this does not happen under dimensional oxidation. Note, however, that the value of $\Delta$ is nonetheless preserved under dimensional reduction and oxidation [5, 6].

Thus far, we have been discussing the single-step oxidation from a domain wall in a particular dimension to a stack of $(D-3)$-branes in one dimension higher. Of course, one can continue the oxidation process further on up. Since the dimensional reduction of supergravity theories is a consistent procedure both for the standard Kaluza-Klein type of reduction and also for the Scherk-Schwarz type of reduction, it follows that all solutions of a given dimensionally-reduced theory can be traced back to the highest dimension in which that theory has its origins. In this paper, our principal focus has been on the massive theories originating from $D=11$ supergravity, and thus we may consider the oxidation of all lower-dimensional domain-wall solutions up to $D=11$. Of course, this only makes sense for those domain walls in lower dimensions that are solutions of the massive supergravities derivable from $D=11$. In particular, we have seen that the dimensional reduction of the massive IIA theory in $D=10$ is inequivalent to all massive theories that can be obtained by reduction from $D=11$, and thus there is no sense in which solutions of the massive IIA theory can be related to those of $D=11$ supergravity.

The 6 -brane solution of the massive supergravity in $D=8$ that we constructed in section 2.1 has the metric $d s_{8}^{2}=H^{1 / 6} d x^{\mu} d x_{\mu}+H^{7 / 6} d y^{2}$. This oxidises to $d s_{9}^{2}=d x^{\mu} d x_{\mu}+H\left(d y^{2}+\right.$ $\left.d z_{3}^{2}\right)$ in $D=9$, and then to $d s_{10}^{2}=H^{-1 / 8} d x^{\mu} d x_{\mu}+H^{7 / 8}\left(d y^{2}+d z_{2}^{2}+d z_{3}^{2}\right)$ in $D=10$. Finally, upon oxidation to $D=11$, it becomes

$$
d s_{11}^{2}=d x^{\mu} d x_{\mu}+H\left(d y^{2}+d z_{2}^{2}+d z_{3}^{2}\right)+H^{-1}\left(d z_{1}+\mathcal{A}_{1}^{(1)}\right)^{2},
$$


where $\mathcal{A}_{1}^{(1)}=m z_{3} d z_{2}$, and the coordinates $x^{\mu}$ span the 7 -dimensional worldvolume of the solution. The harmonic function $H$ remains the same under these oxidation steps, and continues to involve only the original transverse coordinate $y$ as did the starting 6brane in $D=8$. Owing to the Scherk-Schwarz oxidation step, the higher-dimensional solutions have a dependence on $z_{3}$, either in the reduction axion in the $D=9$ case, or in its $D=10$ progenitor $\mathcal{A}_{1}^{(1)}$, or finally in the $D=11$ metric, as one sees in (5.1). All of these oxidised solutions have the same worldvolume Poincaré symmetries as the original 6-brane in $D=8$, but owing to their non-isotropic structure in the transverse space, they do not have interpretations as standard $p$-branes.

In $D=7$, we have seen that there are five massive supergravity theories that can be obtained from $D=11$ supergravity. One of these is the diagonal dimensional reduction of the $D=8$ theory that we discussed in section (2.1). Thus, the oxidation of its domain wall solution to $D=11$ gives the same metric as above, but with the $7^{\text {th }}$ worldvolume coordinate replaced by the compactification coordinate $z_{4}$ :

$$
d s_{11}^{2}=d x^{\mu} d x_{\mu}+d z_{4}^{2}+H\left(d y^{2}+d z_{2}^{2}+d z_{3}^{2}\right)+H^{-1}\left(d z_{1}+\mathcal{A}_{1}^{(1)}\right)^{2} .
$$

The vector potential is again given by $\mathcal{A}_{1}^{(1)}=m z_{3} d z_{2}$, and the coordinates $x^{\mu}$ now span the 6-dimensional worldvolume of the solution, which retains the $d=6$ Poincaré symmetry of the starting solution. The remaining four massive $D=7$ theories that come from $D=11$ are the ones that we constructed in section 2.2. The corresponding four $\Delta=4$ domain walls in $D=7$ oxidise in different fashions, eventually giving the following metrics in $D=11$ :

$$
\begin{array}{ll}
\mathcal{A}_{0}^{(12)}: & d s_{11}^{2}=d x^{\mu} d x_{\mu}+d z_{3}^{2}+H\left(d y^{2}+d z_{2}^{2}+d z_{4}^{2}\right)+H^{-1}\left(d z_{1}+\mathcal{A}_{1}^{(1)}\right)^{2}, \\
\mathcal{A}_{0}^{(13)}: & d s_{11}^{2}=d x^{\mu} d x_{\mu}+d z_{2}^{2}+H\left(d y^{2}+d z_{3}^{2}+d z_{4}^{2}\right)+H^{-1}\left(d z_{1}+\mathcal{A}_{1}^{(1)}\right)^{2}, \\
\mathcal{A}_{0}^{(23)}: & d s_{11}^{2}=d x^{\mu} d x_{\mu}+d z_{1}^{2}+H\left(d y^{2}+d z_{3}^{2}+d z_{4}^{2}\right)+H^{-1}\left(d z_{2}+\mathcal{A}_{1}^{(2)}\right)^{2}, \\
A_{0}^{(123)}: & d s_{11}^{2}=H^{-\frac{1}{3}} d x^{\mu} d x_{\mu}+H^{\frac{2}{3}}\left(d y^{2}+d z_{1}^{2}+d z_{2}^{2}+d z_{3}^{2}+d z_{4}^{2}\right),
\end{array}
$$

where we indicate which axion is used in the Scherk-Schwarz reduction for each case. In the first three metrics given here, the gauge potential is given by $\mathcal{A}_{1}^{(1)}=m z_{4} d z_{2}, \mathcal{A}_{1}^{(1)}=m z_{4} d z_{3}$ and $\mathcal{A}_{1}^{(2)}=m z_{4} d z_{3}$ respectively. The last metric, corresponding to the oxidation of the domain wall solution of the $A_{0}^{(123)}$ massive theory in $D=7$, has a straightforward elevendimensional interpretation as a continuous 4 -volume of 5-branes. The previous cases, which all come from solutions where the axion used in the Scherk-Schwarz reduction originates from the metric, give rise to "twisted" solutions in $D=11$.

The 5-brane solution of the sixth massive $D=7$ supergravity theory, which comes from the Kaluza-Klein dimensional reduction of the massive IIA theory in $D=10$, can be 
oxidised back to the 8-brane [21] of the massive IIA theory, or to a stack of 7-branes in the type IIB theory in $D=10$ [9].

As we saw in section 3 , it is also possible to construct 5 -branes with $\Delta=2$ in $D=7$. These will oxidise back to appropriate intersections of the $\Delta=4$ oxidations described above.

As one descends through the dimensions, similar considerations can be applied to all of the domain-wall solutions to the multitude of massive supergravities. One general feature that is worth remarking upon is that the only values of $\Delta$ that can arise in any of the massive supergravities obtainable from $D=11$ are the set $\Delta=4 / N$, as discussed above in section 3 . In particular, this means that if a massive supergravity theory has a single-scalar truncation with any value of $\Delta$ other than one of these allowed ones, then one can immediately deduce that it cannot come from $D=11$ by Scherk-Schwarz dimensional reduction. Examples of such independent massive supergravities are the gauged supergravities in $D=7$ [22], $D=6$ [23, 24] and $D=5$ [25], and the Freedman-Schwarz gauged theory in $D=4$ [26]. Interestingly, these all have $\Delta=-2$. While it is conceivable therefore that they might all have a common origin in the gauged $D=7$ theory, it must certainly be the case that none of them can come from $D=11$ supergravity by Scherk-Schwarz reduction.

\section{Discussion and conclusions}

In this paper, we have studied the massive supergravity theories that can be obtained by Scherk-Schwarz dimensional reduction from $D=11$ supergravity. This is a generalisation of the usual Kaluza-Klein dimensional reduction, in which at one stage of the step-bystep reduction from eleven to $D$ dimensions, one or more of the axions in the theory in an intermediate dimension are allowed to have a linear dependence on the compactification coordinate $z$, of the form given in (2.7). This generalised ansatz still yields a consistent truncation of the theory, because the axions in question are covered by derivatives everywhere in the Lagrangian, and thus the Lagrangian will be independent of $z$ after substitution of the ansatz. The ansatz has the effect of generating cosmological-type terms after the dimensional reduction, together with mass terms for certain fields in the theory.

We have seen that there are many different massive supergravities that can be obtained by this procedure. This contrasts with the situation for the usual Kaluza-Klein reduction of $D=11$ supergravity, where one obtains just one massless maximal supergravity in each dimension. The reason for this multiplicity of massive theories is that the Scherk-Schwarz reduction step commutes neither with ordinary Kaluza-Klein reduction, nor with U-duality. 
Thus, for example, we obtain a single massive supergravity in $D=8$ by this method, since there is only one axion in $D=9$ supergravity, but in $D=7$ we obtain in total five different massive supergravities. Four of these correspond to the four different axions in $D=8$ that can be used for the Scherk-Schwarz reduction, and the fifth is the theory obtained by ordinary Kaluza-Klein reduction of the massive theory we already constructed in $D=8$. In addition, there is always one further massive maximal supergravity in each dimension $D \leq 10$, corresponding to the massive IIA supergravity [10] and its Kaluza-Klein dimensional reductions. This latter sequence of theories is quite distinct from any of those obtainable from $D=11$ supergravity by the methods used in this paper. In particular, the conjectured duality [9] in $D=8$ of the vertically-reduced 6-brane of massless type IIA supergravity and the diagonally-reduced 8-brane of massive type IIA supergravity seems to be false. It is intriguing, though, that the dimensional reduction of the massive IIA theory to $D=9$ is the same as the theory one obtains by the Scherk-Schwarz reduction of the IIB theory in $D=10$ [9].

The distinguished rôle that the massive type IIA theory appears to play, amongst the set of all massive theories, suggests the possibility that it may have its origins in some more fundamental theory. This would be in a similar spirit to the introduction of M-theory, which is dual to the massless type IIA string, and F-theory, which is dual to the type IIB string. In this second example, the $S L(2, Z)$ symmetry of the type IIB theory can then be understood as coming from the geometry of the 2-torus used to compactify from $D=12$ to $D=10$ [27. Thus we might conjecture the existence of an hypothetical H-theory, whose compactification would give rise to the massive IIA theory in $D=10$. As we have seen, a massive supergravity can be obtained by performing an $S^{1}$ reduction of a theory in one higher dimension, by allowing an axion, or 0-form potential, to depend linearly on the compactification coordinate. The most natural source of such an axion in $D=11$ would be from the dimensional reduction of the metric in a theory in $D=13$. (The existence of a theory in $D=13$ has also been proposed in [28].) Thus we may imagine that there are three fundamental theories, namely $\mathrm{M}, \mathrm{F}$ and $\mathrm{H}$ in $D=11,12$ and 13 , which are the progenitors of the IIA, IIB and massive IIA theories respectively. Just as the IIA and IIB theories compactified on $S^{1}$ are T-duals, so the IIB and massive IIA compactified on $S^{1}$ are T-duals (where in this latter case, the $S^{1}$ compactification of the IIB theory involves a linear dependence of the axion on the compactification coordinate).

There is in fact another way to interpret the Scherk-Schwarz dimensional reduction that we have been carrying out in this paper. Let us suppose that we have a formulation 
of the massless theory in $D+1$ dimensions in which the axion $\chi$ is covered everywhere by a derivative. Since it therefore appears in the Lagrangian only via its 1-form field $F_{D}={ }^{*} F_{1}$, we may rewrite the Lagrangian in a dualised form in terms of the $D$-form field strength $F_{D}={ }^{*} F_{1}$. Upon now performing a standard Kaluza-Klein reduction, in which the potential for $F_{D}$ is re-expressed in terms of $z$-independent $D$-dimensional potentials $A_{D-1}$ and $A_{D-2}$ as in (2.1), the $D$-dimensional Lagrangian will acquire kinetic terms for field strengths $F_{D}$ and $F_{D-1}$ of ranks $D$ and $(D-1)$. If these are then dualised in $D$ dimensions, the first will give rise to a cosmological-type term, and the second will give a 1-form field strength that can be interpreted as the derivative of an axion. This is the same result that we obtained directly by simply performing the Scherk-Schwarz reduction of the axion in $(D+1)$ dimensions. Clearly, this alternative description can also be generalised to the case where more than one axion is covered by derivatives simultaneously, and thus we have a general statement that for each such axion there is a correspondence between a Scherk-Schwarz reduction step on the one hand, and a dualisation, followed by a KaluzaKlein reduction step, followed by a dualisation in the lower dimension. From our point of view, however, it should be emphasised that this alternative description is not completely equivalent: after dualising the 1-form field strength of a 0 -form axion, and performing a Kaluza-Klein reduction, one cannot give a local expression for the higher-dimensional 0form in terms of the lower-dimensional fields. Since we are interested in formulating the dimensional reduction process in such a way that all solutions of the lower-dimensional theories are re-interpreted as solutions of $D=11$ supergravity, it is important that one should be able to give the Kaluza-Klein ansatz for the $D=11$ metric and 3-form potential in terms of the fields of the lower-dimensional theories, and thus dualisations at intermediate stages of the reduction should be avoided.

For the same reason, the potential $A_{3}$ in $D=5$, and the potentials $A_{2}^{(i)}$ in $D=4$, should be kept in these original forms, and should not be reformulated as axions by performing dualisations. Thus these fields will undergo standard Kaluza-Klein reductions to $D=4$ and $D=3$ respectively, giving rise to (non-propagating) $D$-form descriptions of massive supergravities, rather than the dual descriptions with cosmological terms. It is interesting to note that, historically, the first example of a massive supergravity theory was of just such a kind. Specifically, a massive theory in $D=4$ was obtained by performing the KaluzaKlein dimensional reduction of the 4 -form field strength $F_{4}$ in $D=5$, and then dualising the resulting 4 -form in $D=4$ to give a cosmological-type term 29.

It is perhaps worth remarking, in the light of the above discussion, that one can in fact 
reformulate the massive IIA theory in ten dimensions in terms of a 10-form field strength rather than a cosmological-type term [9]. Effectively, the mass parameter of the massive IIA theory now arises as a constant of integration. In particular, this constant of integration can take the value zero, implying that all solutions of the massless IIA theory are also solutions of the 10-form reformulation of the massive IIA theory. Since all of our massive theories that are obtainable from $\mathrm{D}=11$ pass through $D=10$ without yet having performed any Scherk-Schwarz reduction, it follows that they can equally well be viewed as descendants of the massless IIA theory in $D=10$. Then, by virtue of the previous observation, we may say that they are in fact descendants of the 10-form reformulation of the massive IIA theory in $D=10$. In this sense, one may in fact take the point of view that this formulation of the massive IIA theory provides a "universal" theory from which all the lower-dimensional massive theories are derivable. They can of course also therefore be "derived" from the original formulation of the massive IIA theory with a cosmological term, in that those massive theories that we have obtained from $D=11$ can also be obtained from the massive IIA theory by first setting its mass parameter to zero. However, although this gives an algorithm for generating theories, their solutions would not be solutions of the massive IIA theory in its original form, since its mass parameter is a given, fixed quantity, and not an arbitrary integration constant that can take any value including zero.

The above observations do not alter the fact that there exist inequivalent maximallysupersymmetric massive theories in each dimension $D \leq 8$. In particular, the theory that one gets by performing a Scherk-Schwarz reduction on any one axion is inequivalent to the theory one gets by performing the Scherk-Schwarz reduction on any other axion. There are, as we have seen, larger theories with multiple parameters that correspond to performing simultaneous Scherk-Schwarz reductions on more than one axion. However, it is not possible to perform Scherk-Schwarz reductions on all the axions simultaneously, and thus there does not in general exist any single universal theory in a lower dimension that encompasses all of the single-axion reductions at the same time.

\section{Appendix A.}

The dimensional reduction of the bosonic sector of $D=11$ supergravity to $D$ dimensions, in the formalism that we are using in this paper, is given by [11]

$$
e^{-1} \mathcal{L}=R-\frac{1}{2}(\partial \vec{\phi})^{2}-\frac{1}{48} e^{\vec{a} \cdot \vec{\phi}} F_{4}^{2}-\frac{1}{12} \sum_{i} e^{\vec{a}_{i} \cdot \vec{\phi}}\left(F_{3}^{i}\right)^{2}-\frac{1}{4} \sum_{i<j} e^{\vec{a}_{i j} \cdot \vec{\phi}}\left(F_{2}^{i j}\right)^{2}
$$




$$
-\frac{1}{4} \sum_{i} e^{\vec{b}_{i} \cdot \vec{\phi}}\left(\mathcal{F}_{2}^{i}\right)^{2}-\frac{1}{2} \sum_{i<j<k} e^{\vec{a}_{i j k} \cdot \vec{\phi}}\left(F_{1}^{i j k}\right)^{2}-\frac{1}{2} \sum_{i<j} e^{\vec{b}_{i j} \cdot \vec{\phi}}\left(\mathcal{F}_{1}^{i j}\right)^{2}+e^{-1} \mathcal{L}_{F F A}
$$

where $F_{4}, F_{3}^{i}, F_{2}^{i j}$ and $F_{1}^{i j k}$ are the 4-form, 3-forms, 2-forms and 1-forms coming from the dimensional reduction of $\hat{F}_{4}$ in $D=11 ; \mathcal{F}_{2}^{i}$ are the 2-forms coming from the dimensional reduction of the vielbein, and $\mathcal{F}_{1}^{i j}$ are the 1 -forms coming from the dimensional reduction of these 2-forms. The axions that are the focus of our attention in much of this paper are the 0 -form potentials $A_{0}^{i j k}$ and $\mathcal{A}_{0}^{i j}$.

The $(11-D)$ scalar fields denoted by $\vec{\phi}$ are the dilatonic scalars arising at each step in the sequential Kaluza-Klein reduction of the metric. The $\vec{a}$ and $\vec{b}$ vectors appearing in the exponentials are constants characterising the couplings of the dilatonic scalars to the various field strengths. They are given by [11]

$$
\begin{array}{lll} 
& F_{M N P Q} & \text { vielbein } \\
4-\text { form }: & \vec{a}=-\vec{g}, & \\
3-\text { forms }: & \vec{a}_{i}=\vec{f}_{i}-\vec{g} & \\
2-\text { forms }: & \vec{a}_{i j}=\vec{f}_{i}+\vec{f}_{j}-\vec{g}, & \vec{b}_{i}=-\vec{f}_{i}, \\
1-\text { forms : } & \vec{a}_{i j k}=\vec{f}_{i}+\vec{f}_{j}+\vec{f}_{k}-\vec{g}, & \vec{b}_{i j}=-\vec{f}_{i}+\vec{f}_{j}, \\
0-\text { forms : } & \vec{a}_{i j k \ell}=\vec{f}_{i}+\vec{f}_{j}+\vec{f}_{k}+\vec{f}_{\ell}-\vec{g}, & \vec{b}_{i j k}=-\vec{f}_{i}+\vec{f}_{j}+\vec{f}_{k}
\end{array}
$$

where the vectors $\vec{g}$ and $\vec{f}_{i}$ have $(11-D)$ components in $D$ dimensions, and are given by

$$
\begin{aligned}
\vec{g} & =3\left(s_{1}, s_{2}, \ldots, s_{11-D}\right) \\
\overrightarrow{f_{i}} & =(\underbrace{0,0, \ldots, 0}_{i-1},(10-i) s_{i}, s_{i+1}, s_{i+2}, \ldots, s_{11-D}),
\end{aligned}
$$

where $s_{i}=\sqrt{2 /((10-i)(9-i))}$. It is easy to see that they satisfy

$$
\vec{g} \cdot \vec{g}=\frac{2(11-D)}{D-2}, \quad \vec{g} \cdot \overrightarrow{f_{i}}=\frac{6}{D-2}, \quad \overrightarrow{f_{i}} \cdot \overrightarrow{f_{j}}=2 \delta_{i j}+\frac{2}{D-2} .
$$

We have included the dilaton vectors for "0-form field strengths" in (A.2) because these fit into the same general pattern, and because they correspond to the cosmological-type terms arising in the Scherk-Schwarz reductions discussed in this paper.

In general, the field strengths appearing in the kinetic terms acquire Chern-Simons type modifications in the dimensional reduction process, given by 11

$$
\begin{aligned}
& F_{4}=\tilde{F}_{4}-\gamma^{i j} \tilde{F}_{3}^{i} \wedge \mathcal{A}_{1}^{j}-\frac{1}{2} \gamma^{i k} \gamma^{j \ell} \tilde{F}_{2}^{i j} \wedge \mathcal{A}_{1}^{k} \wedge \mathcal{A}_{1}^{\ell}+\frac{1}{6} \gamma^{i \ell} \gamma^{j m} \gamma^{k n} \tilde{F}_{1}^{i j k} \wedge \mathcal{A}_{1}^{\ell} \wedge \mathcal{A}_{1}^{m} \wedge \mathcal{A}_{1}^{n} \\
& F_{3}^{i}=\gamma^{j i} \tilde{F}_{3}^{j}-\gamma^{j i} \gamma^{k \ell} \tilde{F}_{2}^{j k} \wedge \mathcal{A}_{1}^{\ell}-\frac{1}{2} \gamma^{j i} \gamma^{k m} \gamma^{\ell n} \tilde{F}_{1}^{j k \ell} \wedge \mathcal{A}_{1}^{m} \wedge \mathcal{A}_{1}^{n},
\end{aligned}
$$




$$
\begin{aligned}
F_{2}^{i j} & =\gamma^{k i} \gamma^{\ell j} \tilde{F}_{2}^{k \ell}-\gamma^{k i} \gamma^{\ell j} \gamma^{m n} \tilde{F}_{1}^{k \ell m} \wedge \mathcal{A}_{1}^{n}, \\
F_{1}^{i j k} & =\gamma^{\ell i} \gamma^{m j} \gamma^{n k} \tilde{F}_{1}^{\ell m n}, \\
\mathcal{F}_{2}^{i} & =\tilde{\mathcal{F}}_{2}^{i}-\gamma^{j k} \tilde{\mathcal{F}}_{1}^{i j} \wedge \mathcal{A}_{1}^{k}, \\
\mathcal{F}_{1}^{i j} & =\gamma^{k j} \tilde{\mathcal{F}}_{1}^{i k},
\end{aligned}
$$

where the tilded quantities denote the unmodified field strengths given directly by the exterior derivatives of gauge potentials. The matrix $\gamma^{i j}$ is given by

$$
\gamma^{i j}=\left[\left(1+\mathcal{A}_{0}\right)^{-1}\right]^{i j}=\delta^{i j}-\mathcal{A}_{0}^{i j}+\mathcal{A}_{0}^{i k} \mathcal{A}_{0}^{k j}-\mathcal{A}_{0}^{i k} \mathcal{A}_{0}^{k \ell} \mathcal{A}_{0}^{\ell j}+\cdots
$$

The 0 -form potentials $\mathcal{A}_{0}^{i j}$ are defined only for $i<j$, i.e. $\mathcal{A}_{0}^{i j}=0$ for $i \geq j$, and hence it follows that the series (A.7) for $\gamma^{i j}$ terminates after $j-i+1 \leq 11-D$ terms, with $\gamma^{i j}=0$ for $i>j$ and $\gamma^{i j}=1$ for $i=j$.

The term $\mathcal{L}_{F F A}$ in A.1 is the dimensional reduction of the $\tilde{F}_{4} \wedge \tilde{F}_{4} \wedge A_{3}$ term in $D=11$, and is given in lower dimensions by 11

$$
\begin{array}{ll}
D=10: & \frac{1}{2} \tilde{F}_{4} \wedge \tilde{F}_{4} \wedge A_{2}, \\
D=9: & \left(-\frac{1}{4} \tilde{F}_{4} \wedge \tilde{F}_{4} \wedge A_{1}^{i j}-\frac{1}{2} \tilde{F}_{3}^{i} \wedge \tilde{F}_{3}^{j} \wedge A_{3}\right) \epsilon_{i j} \\
D=8: & \left(-\frac{1}{12} \tilde{F}_{4} \wedge \tilde{F}_{4} A_{0}^{i j k}-\frac{1}{6} \tilde{F}_{3}^{i} \wedge \tilde{F}_{3}^{j} \wedge A_{2}^{k}+\frac{1}{2} \tilde{F}_{3}^{i} \wedge \tilde{F}_{2}^{j k} \wedge A_{3}\right) \epsilon_{i j k}, \\
D=7: & \left(-\frac{1}{6} \tilde{F}_{4} \wedge \tilde{F}_{3}^{i} A_{0}^{j k l}+\frac{1}{6} \tilde{F}_{3}^{i} \wedge \tilde{F}_{3}^{j} \wedge A_{1}^{k l}+\frac{1}{8} \tilde{F}_{2}^{i j} \wedge \tilde{F}_{2}^{k l} \wedge A_{3}\right) \epsilon_{i j k l}, \\
D=6: & \left(\frac{1}{12} \tilde{F}_{4} \wedge \tilde{F}_{2}^{i j} A_{0}^{k l m}+\frac{1}{12} \tilde{F}_{3}^{i} \wedge \tilde{F}_{3}^{j} A_{0}^{k l m}+\frac{1}{8} \tilde{F}_{2}^{i j} \wedge \tilde{F}_{2}^{k l} \wedge A_{2}^{m}\right) \epsilon_{i j k l m}, \\
D=5: & \left(\frac{1}{12} \tilde{F}_{3}^{i} \wedge \tilde{F}_{2}^{j k} A_{0}^{l m n}-\frac{1}{48} \tilde{F}_{2}^{i j} \wedge \tilde{F}_{2}^{k l} \wedge A_{1}^{m n}-\frac{1}{72} \tilde{F}_{1}^{i j k} \wedge \tilde{F}_{1}^{l m n} \wedge A_{3}\right) \epsilon_{i j k l m n}, \\
D=4: & \left(-\frac{1}{48} \tilde{F}_{2}^{i j} \wedge \tilde{F}_{2}^{k l} A_{0}^{m n p}-\frac{1}{72} \tilde{F}_{1}^{i j k} \wedge \tilde{F}_{1}^{l m n} \wedge A_{2}^{p}\right) \epsilon_{i j k l m n p}, \\
D=3: & \frac{1}{144} \tilde{F}_{1}^{i j k} \wedge \tilde{F}_{1}^{l m n} \wedge A_{1}^{p q} \epsilon_{i j k l m n p q}, \\
D=2: & \frac{1}{1296} \tilde{F}_{1}^{i j k} \wedge \tilde{F}_{1}^{l m n} A_{0}^{p q r} \epsilon_{i j k l m n p q r} .
\end{array}
$$

\section{Acknowledgement}

K.S.S. would like to thank S.I.S.S.A. and the Yukawa Institute, University of Kyoto, for hospitality during the course of this work.

\section{References}

[1] H. Lü, C.N. Pope and K.S. Stelle, Weyl group invariance and p-brane multiplets, hep-th/9602140, to appear in Nucl. Phys. B. 
[2] C.M. Hull and P.K. Townsend, Unity of superstring dualities, Nucl. Phys. B294 (1995) 196.

[3] M.J. Duff and J.X. Lu, Duality rotations in membrane theory, Nucl. Phys. B347 (1990) 394.

[4] M.J. Duff, P.S. Howe, T. Inami and K.S. Stelle, Superstrings in $D=10$ from supermembranes in $D=11$, Phys. Lett. B191 (1987) 70.

[5] H. Lü, C.N. Pope, S. Sezgin and K.S. Stelle, Stainless super p-branes, Nucl. Phys. B456 (1995) 669.

[6] H. Lü, C.N. Pope and K.S. Stelle, Vertical Versus Diagonal Dimensional Reduction for p-branes, hep-th/9605082, to appear in Nucl. Phys. B.

[7] M.J. Duff and R.R. Khuri, Four-dimensional string/string dulaity, Nucl. Phys. B411 (1994) 473; M.J. Duff, R.R. Khuri, R. Minasian and J. Rahmfeld, New black hole, string and membrane solutions of the four-dimensional heterotic string, Nucl. Phys. B418 (1994) 195.

[8] J. Scherk and J.H. Schwarz, Spontaneous breaking of supersymmetry through dimensional reduction, Phys. Lett. B82 (1979) 60.

[9] E. Bergshoeff, M. de Roo, M.B. Green, G. Papadopoulos and P.K. Townsend, Duality of Type II 7-branes and 8-branes, hep-th/9601150.

[10] L.J. Romans, Massive $N=2 a$ supergravity in ten dimensions, Phys. Lett. B169 (1986) 374.

[11] H. Lü and C.N. Pope, p-brane solitons in maximal supergravities, Nucl. Phys. B465 (1996) 127.

[12] H. Lü, C.N. Pope, E. Sezgin and K.S. Stelle, Dilatonic p-brane solitons, Phys. Lett. B371 (1996) 46.

[13] M. Cvetic, Extreme domain wall — black hole complementarity in $N=1$ supergravity with a general dilaton coupling, Phys. Lett. B341 (1994) 160.

[14] M. Cvetic and H.H. Soleng, Supergravity domain walls, hep-th/9604090.

[15] E. Cremmer and B. Julia, The $N=8$ supergravity theory - the Lagrangian, Phys. Lett. B80 (1978) 48; The SO(8) supergravity, Nucl. Phys. B159 (1979) 141. 
[16] L. Mezincescu, P.K. Townsend and P. van Nieuwenhuizen, Stability of gauged $D=7$ supergravity and the definition of masslessness in (ADS) in seven-dimensions, Phys. Lett. B143 (1984) 384.

[17] M. Cvetic, S. Griffies and S-J. Rey, Static domain walls in $N=1$ supergravity, Nucl. Phys. B381 (1992) 301.

[18] H. Lü and C.N. Pope, $S L(N+1, \mathbb{R})$ Toda solitons in supergravities, hep-th/9607027.

[19] H. Lü and C.N. Pope, Multi-scalar p-brane solitons, hep-th/9512153, to appear in Int. J. Mod. Phys. A.

[20] M.J. Duff, H. Lü and C.N. Pope, The black branes of M-theory, Phys. Lett. B382 (1996) 73.

[21] J. Polchinski and E. Witten, Evidence for heterotic-type I string duality, Nucl. Phys. B460 (1996) 525.

[22] P.K. Townsend and P. van Nieuwenhuizen, Gauged seven-dimensional supergravity, Phys. Lett. B125 (1983) 41.

[23] M.A. Awada and P.K. Townsend, Gauged $N=4 D=6$ Maxwell-Einstein supergravity and 'antisymmetric tensor Chern-Simons' forms, Phys. Rev. D33 (1985) 1557.

[24] L.J. Romans, The F(4) gauged supergravity in six dimensions, Nucl. Phys. 269 (1986) 691.

[25] M. Günaydin, G. Sierra and P.K. Townsend, Vanishing potentials in gauged $N=2$ supergravity: an application of jordan algebras, Phys. Lett. B144 (1984) 41.

[26] D.Z. Freedman and J.H. Schwarz, $N=4$ supergravity theory with local $S U(2) \times S U(2)$ invariance, Nucl. Phys. B137 (1978) 333.

[27] C. Vafa, Evidence for F-theory, Nucl. Phys. B469 (1996) 403.

[28] I. Bars, S theory, hep-th/9607112.

[29] A. Aurilia, H. Nicolai and P.K. Townsend, Hidden constants: the theta parameter of $Q C D$ and the cosmological constant of $N=8$ supergravity, Nucl. Phys. B176 (1980) 509. 